\title{
Involvement of Bicarbonate-Induced Radical Signaling in Oxysterol Formation and Sterol Depletion of Capacitating Mammalian Sperm During In Vitro Fertilization ${ }^{1}$
}

Arjan Boerke, ${ }^{3}$ Jos F. Brouwers, ${ }^{3}$ Vesa M. Olkkonen, ${ }^{4}$ C.H.A. van de Lest, ${ }^{3}$ Edita Sostaric, ${ }^{5}$ Eric J. Schoevers, ${ }^{6}$ J. Bernd Helms, ${ }^{3}$ and Barend M. Gadella ${ }^{2,3,6}$

${ }^{3}$ Department of Biochemistry and Cell Biology, Faculty of Veterinary Medicine, Utrecht University, Utrecht, The Netherlands

${ }^{4}$ Minerva Foundation Institute for Medical Research, Biomedicum Helsinki 2U Helsinki, Finland

${ }^{5}$ Department of Equine Sciences, Faculty of Veterinary Medicine, Utrecht University, Utrecht, the Netherlands

${ }^{6}$ Department of Farm Animal Health, Faculty of Veterinary Medicine, Utrecht University, Utrecht, The Netherlands

${ }^{1}$ This work has been supported by the High Potential Program from Utrecht University.

${ }^{2}$ Correspondence: E-mail: b.m.gadella@uu.nl

\begin{abstract}
This study demonstrates for the first time that porcine and mouse sperm incubated in capacitation media supplemented with bicarbonate produce oxysterols. The production is dependent on a reactive oxygen species (ROS) signaling pathway that is activated by bicarbonate and can be inhibited or blocked by addition of vitamin $\mathrm{E}$ or vitamin A or induced in absence of bicarbonate with pro-oxidants. The oxysterol formation was required to initiate albumin dependent depletion of $30 \%$ of the total free sterol and $>50 \%$ of the formed oxysterols. Incubation of bicarbonate treated sperm with oxysterol binding proteins (ORP-1 or -2) caused a reduction of $>70 \%$ of the formed oxysterols in the sperm pellet but no free sterol depletion. Interestingly, both ORP and albumin treatments led to similar signs of sperm capacitation: hyper-activated motility, tyrosin phosphorylation, aggregation of flotillin in the apical ridge area of the sperm head. However, only albumin incubations led to high in vitro fertilization rates of the oocytes whereas the ORP-1 and -2 incubations did not. A pretreatment of sperm with vitamin $E$ or A caused reduced in vitro fertilization rates with $47 \%$ and $100 \%$, respectively. Artificial depletion of sterols mediated by methyl-beta cyclodextrin bypasses the bicarbonate ROS oxysterol signaling pathway but resulted only in low in vitro fertilization rates and oocyte degeneration. Thus bicarbonate induced ROS formation causes at the sperm surface oxysterol formation and a simultaneous activation of reverse sterol transport from the sperm surface which appears to be required for efficient oocyte fertilization.
\end{abstract}

\section{INTRODUCTION}

Before the sperm cell can enter the oocyte it first needs to be activated. This activation process has been described extensively [1,2]. One of the hallmarks of this activation process (also termed capacitation) is the increased binding affinity of the sperm cell to the zona pellucida (ZP; the extracellular matrix) of the oocyte. The main components thought to be responsible for sperm capacitation are bicarbonate and $\mathrm{Ca}^{2+}$ levels. Both ions induce signaling cascades after elevated levels inside the sperm cell [3]. Besides these two ions, albumin acts in synergy by mediating efflux of sterols from the sperm's surface $[4,5]$. In boar sperm (one of our model species in this study) albumin is thought to selectively extract free sterols -like cholesterol and 
desmosterol - from the sperm cells [5-7]. Albumin is rather specific for causing sterol depletion from the sperm surface as phospholipids and glycolipid levels remain unaltered [1,2]. Given the high hydrophobicity of cholesterol compared to the other -membrane bilayer preferring- lipid classes, it -in theory-against its physical properties is preferentially removed from the sperm surface. Normally albumin is involved in the transport of free fatty acid in the circulation from donor to acceptor tissue [8]. One of the mechanisms, that could play a role in sterol depletion, is the involvement of an active cholesterol transporter in providing free cholesterol to the hydrophobic pocket of albumin (as hypothesized previously [3]). Another option is that sterols can be oxidized and that their oxidation products (which are more hydrophilic) can be extracted by albumin (observed in bovine sperm [4]) or can facilitate an oxysterol dependent scavenger sensitive transport of free sterols to albumin [9]. Inclusion of albumin to in vitro capacitation media as well as the concomitant depletion of sterols by albumin has been shown to be of fundamental importance to achieve in vitro fertilization [5].

Sterol oxidation in sperm

In bovine sperm it has recently been demonstrated that sperm capacitation leads to the formation of oxysterols which are preferentially extracted by albumin [4]. The formation of oxysterols is believed to be initiated by reactive oxygen species (ROS) as it can be induced by tertbutylhydroperoxide [4]. The role of ROS in sperm physiology is ambivalent: On one hand mild ROS formation is reported to be relevant for oxidation mediated sperm signaling events that are involved in sperm capacitation $[6,7]$. On the other hand higher ROS formation rates are reported to be damaging for the sperm cell $[10,11]$.

Sterols and lipid-ordered micro-domain formation on the sperm cell

During in vitro capacitation a lateral rearrangement of the sperm surface sterols has been shown to be dependent on bicarbonate and this rearrangement preceded and was required for albumin mediated sterol depletion [3]. This lateral rearrangement coincides with higher membrane fluidity characteristics of the remaining non-raft membrane area, in which lower sterol levels are detected compared to the apical area of the sperm head membrane where raft aggregation takes place $[2,10,12]$. When albumin and bicarbonate together are used to capacitate sperm, this causes a partial depletion of sterols ( $>15 \%$ of total sterol and depletion was only from the nonraft membrane area [2]). The treatment also leads to the aggregation of lipid-ordered membrane micro-domains at the apical ridge area of the sperm surface in multiple species. Several groups [13] independently established that the detergent resistant membrane fraction not only contains raft markers such as caveolin, flotillin, gangliosides, sterols, sphingolipids (for reviews see [14, 15]) but also is very highly enriched in cumulus- and/or zona binding proteins [16] and in proteins involved in docking and priming of the sperm plasma membrane with the outer acrosome membrane $[17,18]$. These surface redistributions are, therefore, considered to be preparative steps for interaction of sperm with the zona pellucida and/or cumulus layer of the oocyte. This binding and induction of the acrosome reaction enables the sperm to reach the oolemma where eventual fertilization can take place of the oocyte (for review see [19]).

\section{Sterol oxidation and sperm capacitation}

The relationship between capacitation of porcine sperm and oxysterol formation was therefore investigated in the present study with the rationale to see whether this formation could attribute to the depletion of sterols from the capacitating sperm surface [20]. To this end we studied whether incubation of sperm with oxysterol binding proteins could induce in vitro capacitation of 
porcine sperm and possibly could result in sperm capable of fertilizing oocytes in vitro in albumin free media. Sperm were, therefore, capacitated in the absence of albumin but in the presence of two recombinant oxysterol binding protein related proteins (ORP-1 and ORP-2) [21]. Both ORPs have specific binding pockets for oxysterols and are able to transport bound oxysterols from a donor membrane towards specific acceptor membranes. The ORP induced effects were compared to methyl $\beta$ cyclodextrin (MBCD) and to delipidated bovine serum albumin (BSA) mediated sterol extraction in capacitating sperm. The role of bicarbonatedependent oxysterol formation and the subsequent (oxy)sterol depletion in the induction of sperm capacitation in vitro, as well as the modulatory role of pro- and anti-oxidants on these effects are discussed.

\section{MATERIALS AND METHODS}

Animal experiments

The Institutional Animal care and Use Committee of Utrecht University approved this study.

Protein expression of ORP-1 and ORP-2

Plasmid expression vectors for ORP-1s or ORP-2 were used for protein production as previously described [21]. Briefly, glutathione S-transferase (GST) fusion proteins of ORP-2 and ORP-1 were produced in E. coli BL21 and purified over glutathione sepharose 4B (GE Healthcare, Amersham, Buckinghamshire, United Kingdom) columns as described by the manufacturer's instructions. Protein concentrations of purified ORP-1 and ORP-2 were determined by the Bradford assay (Coomassie Plus, Pierce, Rockford, IL, USA) according to manufacturer's instruction. Purity of ORP-1 and ORP-2 protein preparations was analyzed on sodium dodecyl sulfate polyacrylamide gels stained with Coomassie brilliant blue [22].

\section{Sperm incubations}

Ejaculates were collected from boars with proven fertility at Varkens KI Nederland (Deventer, the Netherlands), a commercial enterprise producing insemination doses for pig artificial insemination (AI) for sow herds. Freshly ejaculated sperm was filtered through gauze to remove gelatinous material and subsequently diluted and washed in HEPES buffered saline $(137 \mathrm{mM}$ $\mathrm{NaCl}, 2.5 \mathrm{mM} \mathrm{KCl}, 20 \mathrm{mM}$ HEPES, pH 7.4; HBS). Mouse sperm was aspirated from cauda epididymi of wild type mice (strain B6129SF2/J; stock number 101045 Jackson Laboratories, Bar Harbor, ME, USA). Next, sperm were washed through a discontinuous Percoll (GE, Healthcare, Diegem, Belgium) gradient of $70 \%(\mathrm{v} / \mathrm{v})$ and $35 \%(\mathrm{v} / \mathrm{v})$ as described [3]. All solutions were made iso-osmotic $(290-310 \mathrm{mOsm} / \mathrm{kg})$ with $\mathrm{HBS}$ at $23^{\circ} \mathrm{C}$. Percoll layers were discarded and sperm pellets were resuspended at a final concentration of 100 million sperm cells $/ \mathrm{ml}$ in Hepes buffered Tyrodes media (120 mM NaCl, $21.7 \mathrm{mM}$ Lactate, $20 \mathrm{mM}$ Hepes, 5 $\mathrm{mM}$ glucose, $3.1 \mathrm{mM} \mathrm{KCl}, 2.0 \mathrm{mM} \mathrm{CaCl}_{2}, 1.0 \mathrm{mM}$ pyruvate, $0.4 \mathrm{mM} \mathrm{MgSO}_{4}, 0.3 \mathrm{mM}$ $\mathrm{NaH}_{2} \mathrm{PO}_{4} ; 300 \mathrm{mOsm} / \mathrm{kg}$, $\mathrm{pH} 7.4$; HBT condition further called -Bic) or supplemented with 15 $\mathrm{mM} \mathrm{NaHCO} 3$, equilibrated with $5 \% \mathrm{CO}_{2}$ in humidified atmosphere (further refereed to as $+\mathrm{Bic}$ ). Similarly sperm were incubated - Bic or + Bic media supplemented with (i) $0.3 \% \mathrm{w} / \mathrm{v}$ bovine serum albumin (defatted fraction V, Boehringer Mannheim, Almere, the Netherlands; BSA) or (ii) with $4-16 \mu \mathrm{g} / \mathrm{ml}$ recombinant ORP-1 or ORP-2 or (iii) with $0.5-10 \mathrm{mM}$ methyl-ßcyclodextrin (MBCD). All +Bic conditions were incubated with open vials in $5 \% \mathrm{CO}_{2}$ atmosphere for $2 \mathrm{hrs}$ at $38.5^{\circ} \mathrm{C}$. All -Bic conditions were incubated in airtight vials for $2 \mathrm{hrs}$ at 
$38.5^{\circ} \mathrm{C}$ in a water bath. In some cases sperm incubations were carried out in the presence of either $0.5 \mathrm{mM}$ vitamin E (alpha-tocopherol, Sigma-Aldrich, St. Louis, MO, USA); or $0.5 \mathrm{mM}$ vitamin A (retinol, Sigma-Aldrich, St. Louis, MO, USA); or in the presence of a pro-oxidant mix containing $0.2 \mathrm{mM} \mathrm{FeSO}_{4}$ and $1 \mathrm{mM}$ ascorbate; or in the presence of $30 \mu \mathrm{M} 3$ morpholinosydnonimine hydrochloride (SIN-1, Sigma Aldrich, St. Louis, MO, USA) for the induced formation of peroxynitrite [20,23].

\section{Lipid extraction and mass spectrometry}

After incubation, lipids of 200 million sperm cells were extracted according to the method of Bligh and Dyer (see [4]). Lipids were dried under nitrogen, redissolved in chloroform $/ \mathrm{methanol}$ $(1 / 9 ; \mathrm{v} / \mathrm{v})$ and a fraction corresponding to approximately 1 million cells was subjected to reverse phase chromatography on a $150 \mathrm{~mm}$ x $3 \mathrm{~mm}$ Kinetex $2.6 \mu \mathrm{m}$ column (Phenomenex, Torrance, CA), using isocratic elution (methanol/acetonitrile/2-propanol/chloroform; 90/90/8.5/1.5; $\mathrm{v} / \mathrm{v} / \mathrm{v} / \mathrm{v})$. The column effluent was introduced into an Atmospheric Pressure Chemical Ionization source of a 4000Qtrap mass spectrometer (AB Sciex, Foster City, CA) operated under multiple reaction monitoring mode. Eluting peaks were identified and quantified based on comparison of retention time and product ion spectra with authentic standard as described previously [4]. Briefly, a dose response curve was made of 50 fmole-100 pmole levels of oxysterol standards and of cholesterol and desmosterol that were injected into the same reverse phase column elution. The integrated detector response (linear to the entire concentration range) was used to calculate the amounts of each individual (oxy)sterol species form sperm extracts that were after injection eluting from the reverse phase column (for more experimental details see [4]).

\section{Western-blot immunodetection of tyrosine phosphorylation}

After incubation, a total of 2 million sperm cells were resuspended in $25 \mu \mathrm{l}$ of lithium dodecyl sulfate loading buffer (NuPAGE, Invitrogen, Carlsbad, CA, USA) in the presence of $0.1 \mathrm{M}$ dithiothreitol and heated for 10 minutes at $95^{\circ} \mathrm{C}$. Subsequently, solubilized proteins were loaded and separated on a $12 \%$ polyacrylamide gel and run at $40 \mathrm{~mA}$ for $45 \mathrm{~min}$. Subsequently, proteins were blotted onto nitrocellulose paper (Protran BA85, Whatman, Dassel, Germany) at 60V for $1.5 \mathrm{hrs}$. In order to prevent aspecific binding of antibodies used later in the blotting procedure the nitrocellulose paper with blotted proteins was first incubated in blocking buffer (Tris $25 \mathrm{mM}$, $\mathrm{NaCl}, \mathrm{pH} 7.4$, with $0.5 \%$ Tween, TBS-Tween $0.5 \%$ ) for 10 minutes at room temperature. Subsequently, the nitrocellulose paper with the blotted and blocked proteins was incubated with $1 \%$ BSA TBS-Tween $0.05 \%$ for 1 hour at room temperature. After this step the nitrocellulose paper, with the blotted proteins, was incubated in $0.1 \%$ BSA TBS-Tween $0.05 \%$ supplemented with a mouse monoclonal antibody raised against phosphotyrosine residues (PY20; Becton Dickinson Transduction laboratories, Franklin Lakes, NJ, USA) at a final concentration of 1 $\mu \mathrm{g} / \mathrm{ml}$ overnight at $4{ }^{\circ} \mathrm{C}$. The resulting nitrocellulose paper with blotted proteins was washed six times with 10 minutes per washing step in TBS. After this washing the nitrocellulose was placed into TBS-Tween $0.05 \%$ containing $0.5 \mu \mathrm{g} / \mathrm{ml}$ monoclonal goat-anti mouse antibody conjugated with horse radish peroxidase (Nordic Immunology, Tilburg, the Netherlands) for 90 minutes at 4 ${ }^{\circ} \mathrm{C}$. The resulting nitrocellulose paper with blotted and immunolabeled proteins were washed as described before for removal of unbound PY20 above. Protein bands indirectly immunolabeled with horse radish peroxidase were visualized by chemiluminescence for 5 minutes (enhanced chemiluminescence (ECL) detection kit; Supersignal West Pico, Pierce, Rockford, IL, USA) and captured on a Molecular Imager Chemidoc XRS from Biorad Laboratories (Hercules, CA, USA). 
Immunofluoresence detection of tyrosine phosphorylation and of flotillin

After incubation, 200 million sperm cells were subsequently spun down at $600 \mathrm{~g}$ and resuspended in $1 \mathrm{ml}$ and fixed in 2\% paraformaldehyde in phosphate buffered saline (PBS; $137 \mathrm{mM}$ $\mathrm{NaCl}, 2.7 \mathrm{mM} \mathrm{KCl}, 4.3 \mathrm{mM} \mathrm{Na}_{2} \mathrm{HPO}_{4}, 1.47 \mathrm{mM} \mathrm{KH}_{2} \mathrm{PO}_{4}, \mathrm{pH}$ 7.4) at room temperature for 10 minutes. The fixative was removed by three washing steps and centrifugation $(600 \mathrm{~g})$ steps, the final pellet was resuspended with PBS at original concentration. The washed sperm cells were then smeared on Superfrost (Microm International $\mathrm{GmbH}$, Walldorf, Germany) glass slides and dried for 5 minutes at $37{ }^{\circ} \mathrm{C}$ and subsequently placed in $-20^{\circ} \mathrm{C}$ methanol both for 1 minute for full permeabilization of membranes to ensure optimal intracellular immunolabeling. The immobilized and permeabilized sperm cells were then incubated in blocking buffer (PBS containing $1 \%$ BSA and $0.05 \%$ Tween 20 ) for 1 hour in a humid chamber at $37^{\circ} \mathrm{C}$. After this step, cells were incubated in buffer containing $0.1 \%$ BSA and $0.05 \%$ Tween 20 and either supplemented with $5 \mu \mathrm{g} / \mathrm{ml}$ PY-20 antibody or $10 \mu \mathrm{g} / \mathrm{ml}$ mouse monoclonal anti-flotillin-1 (Becton Dickinson Transduction laboratories; Franklin Lakes, NJ, USA), for $1 \mathrm{hr}$ at $37^{\circ} \mathrm{C}$ in a humidified chamber. Unbound antibodies were removed by gently washing the Superfrost glasses with $3 \mathrm{ml}$ of PBS containing $0.1 \%$ BSA and $0.05 \%$ Tween 20 . The resulting specimen on Superfrost glass slides was subjected to $2 \mu \mathrm{g} / \mathrm{ml}$ monoclonal rabbit anti mouse antibody conjugated with Alexa-488 TM (Invitrogen; Carlsbad, CA, USA) for labelling PY-20 or flotillin1 for 2 hours at 37 degrees in a humidified chamber. After immunolabeling the non-bound antibody conjugates with Alexa-488 TM were removed by three subsequent washing steps with PBS containing $0.1 \%$ BSA and $0.05 \%$ Tween 20 and finally rinsed with PBS and mounted in Fluorsave (Calbiochem, San Diego, CA, USA) and air-tight sealed with nail-polish. Samples were examined with a Nikon Eclipse Ti microscope (Tokyo, Japan) equipped with a mercury lamp and appropriate filters at a minimum magnification of 40x.

\section{Motility assessment of incubated sperm}

After sperm was incubated in HBT media sperm motility was measured by the Spermvision computer assisted sperm analysis (CASA; Minitube GmbH, Tiefenbach, Germany) system for 2 hours. Sperm motility measurements were performed in this system by using $20 \mu \mathrm{m}$ deep Leja-4 chambers (Leja Products B.V.; Nieuw Vennep, the Netherlands). Standard Instrument settings of SpermVision Version 3.0 were used for the analysis of motility, as used by the artificial insemination center (Varkens KI, Deventer) [24]. For mouse sperm the percentage of cells with vigorous lateral head displacement movement was counted.

\section{In vitro fertilization (IVF)}

Ovaries were collected from the slaughterhouse material of adult sows (VION, Groenlo, the Netherlands). The 3-6 mm follicles from individual ovaries were aspirated to retrieve cumulusoocyte complexes (COCs). The COCs were individually selected based using criteria previously described [25]. Subsequently, the selected COCs were matured in vitro (IVM) [25]. COCs were collected in HEPES buffered M199 (Gibco Laboratories Inc., Grand Island, NY, USA) and washed in pre-equilibrated M199 supplemented with $2.5 \mathrm{mM} \mathrm{NaHCO}_{3}, 0.1 \%(\mathrm{w} / \mathrm{v})$ polyvinylpyrrolidone, $100 \mu \mathrm{M}$ cysteamine, $75 \mu \mathrm{g} / \mathrm{ml}$ potassium penicillin $\mathrm{G}$ and $50 \mu \mathrm{g} / \mathrm{ml}$ streptomycin sulfate (oocyte maturation medium; OMM). The selected COCs were cultured for 22 hours in humidified atmosphere in air with $5 \% \mathrm{CO}_{2}$ at $38^{\circ} \mathrm{C}$ in OMM supplemented with 0.05 $\mathrm{IU} / \mathrm{ml}$ recombinant human FSH (rhFSH, Organon, Oss, the Netherlands). Subsequently, the COCs were transferred into OMM without rhFSH for another 22 hours. This second step is 
needed for the oocytes to reach the final meiosis stage II of maturation (the stage they in vivo reach at ovulation). The fully matured oocytes were denuded (removal of cumulus cells) and transferred into an in vitro fertilization medium $(113.1 \mathrm{NaCl}, 3 \mathrm{mM} \mathrm{KCl}, 20 \mathrm{mM}$ Tris, $11.0 \mathrm{mM}$ Glucose, $1.0 \mathrm{mM}$ Caffeine, $7,5 \mathrm{mM} \mathrm{CaCl}_{2}, 5.0 \mathrm{mM}$ Na-pyruvate, either supplemented with $0.1 \%$ (w/v) BSA, $4 \mu \mathrm{g} / \mathrm{ml} \mathrm{ORP-1} \mathrm{or} 4 \mu \mathrm{g} / \mathrm{ml}$ ORP-2. Oocytes were equilibrated in humidified atmosphere in air with $5 \% \mathrm{CO}_{2}$ at $38{ }^{\circ} \mathrm{C}$ for at least 1 hour before adding boar sperm. The sperm was washed through Percoll as described above and diluted to a concentration of $10^{5} \mathrm{cell} / \mathrm{s} / \mathrm{ml}$ in the same IVF medium as the oocytes. Next, depending on the amount of oocytes (20-30) per group 20-30 $\mu$ of this sperm suspension was added to the IVF media that already contained the oocytes. The resulting IVF media contained a total of 1000 sperm cells per oocyte. Subsequently, the oocytes and sperm cells were incubated in a humidified atmosphere in air with $5 \% \mathrm{CO}_{2}$ at 38 ${ }^{\circ} \mathrm{C}$ for 24 hours. Next, the oocytes were washed in PBS with $0.1 \%$ BSA and fixed in $4 \%$ formaldehyde in PBS for a minimum of 1 hour. After fixation oocytes were labeled with $1 \mu \mathrm{g} / \mathrm{ml}$ Sytox Green (Molecular Probes, Invitrogen, Leiden, the Netherlands) in PBS for 5 minutes to label the chromatin. Subsequently the fertilization rate of the oocytes were scored and for unfertilized oocytes, they were scored for MII maturation phase rate and for the rate of cells that did not develop further then the MI maturation stage, or even the germinal vesicle stage (GV) were scored as well as the rates of oocytes that were degenerated during this IVF procedure.

\section{$I V F$ in the presence of $M B C D$}

To determine the fertilization rate of sperm submitted to $0.5-10 \mathrm{mM}$ MBCD the standard IVF procedure was followed as described above although all IVF procedures were performed in absence of BSA. A control IVF experiment was performed on the same collection batch of follicles.

\section{IVF in the presence of antioxidants}

Washed sperm cells were pretreated for 30 minutes with $0.5 \mathrm{mM}$ vitamin A or $0.5 \mathrm{mM}$ vitamin $\mathrm{E}$ (each with a final ethanol concentration of $0.1 \mathrm{vol} \%$ ). A control sample was pretreated for 30 minutes with 0.1 vol $\%$ ethanol without anti-oxidants. The pretreated sperm samples where then diluted 1:10 for further IVF experiments and further processed as described above.

\section{Determination of the sperm-zona interaction at IVF conditions}

After all IVF treatments, individual oocytes were, after extensive washing of each individual oocyte, scored for the amount of sperm cells which had firm interaction with the surrounding zona pellucida. This was determined by using the Sytox Green immunofluorescence and bright field view on a Leica TCS SP2 confocal system (Leica Microsystems, GmbH, Wetzlar, Germany) equipped with a $488 \mathrm{~nm}$ laser using the laser power and acquisition settings at a submaximal pixel value.

\section{Measurement of acrosome integrity}

The acrosome integrity of incubated sperm was measured after 30 and 120 minutes by flow cytometry. Sperm cells were stained directly from the different incubations conditions diluted and supplemented with $1 \mu \mathrm{g} / \mathrm{ml}$ peanut agglutinin conjugated to FITC (PNA-FITC; EY Laboratory, San Mateo, Canada) to distinguish acrosomal reacted cells and with the membrane impermeable vital stain propidium iodide (PI) (final concentration $25 \mathrm{nM}$ ). After gentle homogenizing the sperm suspension, sperm were analyzed on a FACS Calibur flow cytometer 
equipped with a $100 \mathrm{~mW}$ argon laser (Becton Dickinson, San Jose, CA, USA). At the wavelength of $488 \mathrm{~nm}$ sperm cells were excited and the FITC and PI -emission intensity was detected in the logarithmic mode of FL-1 (530/30 nm band pass filter) and FL-3 (620 nm long pass filter). The forward and sideway scatter (FSC and SSC) was detected in the linear mode and sperm specific events were gated for further analysis. The resulting 2 dimensional dot plots represented 10,000-gated events and were made with FL-1 data expressed on the X-axis and FL-3 data on the y-axis. The amount of cells positively stained with one or both fluorescent dyes were scored using quadrant analysis in Win MDI software (Version 2.8 J. Trotter, freeware). The percentage of sperm that showed high intensities for FL-1 channel were regarded as acrosome reacted. Sperm samples were also stained for PNA-FITC for microscopic discrimination of acrosome intact and acrosome reacted status. Briefly, after fixation in $2 \%(\mathrm{w} / \mathrm{v})$ paraformaldehyde for 15 minutes at room temperature, sperm cells were spun down at $600 \mathrm{~g}$ and washed in PBS 3 times. Subsequently, sperm cells were smeared on superfrost glass slides and dried at $37{ }^{\circ} \mathrm{C}$. Next, sperm cells were incubated with $100 \mu \mathrm{g} / \mathrm{ml}$ PNA-FITC for 30 minutes at 37 ${ }^{\circ} \mathrm{C}$ in a humid chamber. After this, sperm cells were rinsed with PBS and mounted in Fluorsave (Calbiochem, San Diego, California, USA) and air-tight sealed with nail-polish. Samples were examined with a Nikon Eclipse Ti microscope (Tokyo, Japan) equipped with a mercury lamp and appropriate filters at a minimum magnification of 40x. Sperm cells with no fluorescence at the acrosome region and with visible apical ridges were considered acrosome intact, whereas, sperm cells with PNA-FITC labeled acrosome regions and no visible apical ridge were considered acrosome reacted.

\section{RESULTS}

Bicarbonate induces a ROS signaling dependent oxidation of cholesterol, which can be blocked by vitamin $E$ or vitamin $A$.

The possible oxidation effects of bicarbonate on free sterols in porcine sperm cells by incubating Percoll washed sperm in the absence of sterol depleting agents were examined. Incubation of Percoll washed sperm cells for 2 hours in albumin free media that were supplemented with bicarbonate induced the formation of oxysterols (see Fig. 1). The levels of oxysterols increased approximately 10-fold, from approx. 0.047 mole $\%$ of total sterol (equivalent of 0.062 pmoles oxysterols per million sperm in absence of bicarbonate; -Bic-BSA) to approx. 0.49 mole $\%$ of total sterol (equaling 0.74 pmoles oxysterols per million sperm in presence of bicarbonate; + BicBSA). The formation of all types of oxysterols was nearly completely inhibited when $0.5 \mathrm{mM}$ vitamin $\mathrm{E}$ or $0.5 \mathrm{mM}$ vitamin A was present during the incubations (see Fig. 1). On the other hand when sperm were incubated in the absence of bicarbonate but with added pro-oxidants ( $\mathrm{FeSO}_{4}$ in the presence of ascorbate) similar oxysterol formation was observed when compared to bicarbonate (Fig. 1), while SIN-1 (stimulates peroxynitrite formation) did not cause any oxysterol formation (Fig. 1). The oxysterol species formed after bicarbonate incubation are depicted in the mass spectrometry graphs (Fig. 2) clearly depicting the formation of 7- and 25hydroxycholesterols, 7- and 22- ketocholesterols and 5,6 $\alpha$ - and $\beta$-epoxycholesterols. Note that the intensity of ions detected as depicted in Fig. 2 are corrected for (oxy)sterol specific ion response curves as described previously and thus the peak height/signal is not necessarily proportional to the amount of oxysterols present [4]. The relative amounts of oxysterol species out of the total amounts of oxysterols recovered from sperm are depicted in Fig. 3 and from these data the following findings were derived: (i) In mouse and porcine sperm the amount of oxysterols formed in sperm incubated with bicarbonate or with pro-oxidants (only tested for 
porcine sperm Fig. 1) were similar to the levels depicted in Fig. 1 for +Bic-BSA and for -BicBSA+pro-oxidants (10-fold when compared to-Bic-BSA; see Table 1). (ii) In mouse and porcine sperm the presence of BSA did not change the amount of oxysterols formed compared to the absence of BSA (see Fig. 1 and Table 1). (iii) The amount and composition of oxysterols formed in sperm incubated with bicarbonate and vitamin E supplementation did not differ from control cells (Figs. 1 and 3). (iv) The relative contribution of 7-ketocholesterol increased and 7hydroxycholesterols decreased in the presence of bicarbonate or after inclusion of pro-oxidants (Fig. 3). (v) Interestingly, in the presence of albumin ( $+\mathrm{BSA}+\mathrm{Bic})$ a relative low proportion of $5,6 \alpha$-epoxycholesterol and a much higher proportion of 5,6 $\beta$-epoxycholesterol and 7ketocholesterol as well as more pronounced decrease in 7-hydroxycholesterols were detected when compared to + Bic-BSA. This probably relates to the fact the oxysterols formed actively exchange between the sperm membrane and albumin which may affect interspecies conversions between the sperm surface and the extracellular albumin (both detected in mouse and porcine sperm; see Table 2).

Vitamin $E$ and $A$ inhibit the induction of hyper-activated sperm motility Bicarbonate and albumin have been described to synergistically induce hyper-activated sperm motility [26] and we show here that they also induce oxysterol formation while vitamin $\mathrm{E}$ and vitamin A inhibited the formation of oxysterols (Figs. 1 and 3). Thus we tested whether sperm hyper-activated sperm motility could be inhibited by vitamin E and vitamin A. About $10 \%$ of control (-Bic-BSA; Fig. 4A) sperm showed hyper-activated sperm motility, whereas incubation for 2 hours in presence of bicarbonate (+Bic-BSA) induced signs of hyper-activated sperm motility as shown with CASA measurements in approximately $20 \%$ of the sperm cells (Fig. 4A). Indeed a synergistic effect was seen when bicarbonate and albumin (+Bic+BSA) were added: Under these conditions $45 \%$ of the sperm showed hyperactive motility (Fig. 4A). In the presence of vitamin $\mathrm{E}$ or vitamin A hyperactivation of the motility by bicarbonate and by the combination of bicarbonate and albumin was completely abolished (Fig. 4A). In presence of BSA a higher proportion of sperm cells showed signs of hyper-activated sperm motility (45\%; see Fig. 4A). When sperm cells were incubated in the presence of vitamin $E$ the induction of hyper-activated sperm motility, in the presence of bicarbonate alone, was completely inhibited. Likewise inclusion of vitamin $\mathrm{E}$ to the in vitro fertilization mimicking medium containing both BSA and bicarbonate also showed a severe but not complete inhibition of hyper-activated sperm motility (Fig. 4A). For the regulation of hyperactivated sperm motility (on tyrosine phosphorylation) similar results were found in mouse sperm which could be inhibited by vitamin $\mathrm{E}$ or with vitamin A (see Table 3).

Vitamin E and A reduce sperm-zona binding and in vitro fertilization Since vitamin $E$ and vitamin A both inhibited the bicarbonate dependent oxysterol formation and hyperactivated motility response to bicarbonate (in presence or absence of albumin) we tested whether the sperm binding properties to the zona pellucida and/or in vitro fertilization rates were inhibited or not. We chose to first entrap high levels $(0.5 \mathrm{mM})$ of hydrophobic antioxidants into the plasma membrane of sperm which followed by a 10 fold dilution in antioxidant free IVF medium and perform the IVF experiment for 24 hours. In this way only sperm and not the oocyte had been in direct contact to high levels of membrane antioxidants. The effective vitamin concentration during the IVF incubation available for the oocytes was $<50 \mu \mathrm{M}$. Pretreatment of sperm in $0.1 \%$ ethanol without antioxidants and 1:10 dilution in IVF medium 
was used as internal control. In all treatments the oocytes did not show significant signs of degeneration (in all cases $<10 \%$ ). The pretreatment of sperm for 30 minutes in $0.1 \%$ ethanol (either without antioxidants or with either $0.5 \mathrm{mM}$ vitamin $\mathrm{A}$ or $0.5 \mathrm{mM}$ vitamin $\mathrm{E}$ ) did not affect sperm viability (for mouse similar findings are represented in Table 3 ). In fact sperm that interacted with the zona pellucida remained motile even after $24 \mathrm{~h}$ of IVF incubations. As is depicted in Fig. 4B a reduction of $>60 \%$ in sperm binding was noted after a pretreatment of sperm with $0.5 \mathrm{mM}$ vitamin A and a 35\% reduction in sperm binding was detected after a pretreatment with $0.5 \mathrm{mM}$ vitamin E. A considerable variety in amount of sperm bound per oocyte was found for each individual ejaculated producing boar tested (Fig. 4B). Remarkably, the amount of sperm bound to the zona pellucida of unfertilized oocytes (M2) monospermic fertilized oocytes (Mono) and polyspermic fertilized oocytes (Poly) was not different (Figure 4B). In line with the larger inhibitory effect of vitamin A when compared to vitamin E on spermzona binding were their respective effects on inhibition of IVF rates. When sperm were pretreated for 30 minutes with $0.5 \mathrm{mM}$ vitamin E prior to IVF a reduction of approximately $50 \%$ in fertilization rates were observed while pretreatment with $0.5 \mathrm{mM}$ vitamin A completely blocked fertilization (Fig. 4C). Although the vitamin E effect was significant $(\mathrm{P}<0.05)$ the inhibitory effect was highly variable between each of the 11 boars tested (Fig. 4C). In line with the absence of sperm and oocyte deterioration was the finding that there was no different correlation between the ratio of monospermic and polyspermic fertilized oocytes after vitamin $\mathrm{E}$ treatment. This shows that the diluted vitamin E concentrations $(0.05 \mathrm{mM})$ during the IVF incubation were not affecting the oocyte's receptivity for sperm.

\section{Effects of oxysterol binding proteins on sperm motility}

Recombinant oxysterol binding proteins ORP-1 and ORP-2 were produced and purified as described in materials and methods (purity was checked in silver stained gels see lane 4 in Figure 5A). The effects of varying concentrations (4-16 $\mu \mathrm{g} / \mathrm{ml})$ of ORP-1 and ORP-2 to sperm suspensions were examined. In the presence of bicarbonate but in the absence of albumin (negative control + Bic-BSA), only $20 \%$ of sperm had hyperactivated motility (Fig. $5 \mathrm{~B}$ ) while in presence of albumin ( + Bic + BSA) about $45 \%$ of sperm showed hyperactivated motility patterns (Fig 5B). Both ORP-1 and ORP2 induced a very strong hyperactivated motility in response to the sperm with highest effects in $55 \%$ of sperm already at the lowest dose tested of $4 \mu \mathrm{g} / \mathrm{ml}$; Fig. 5B).

Effects of oxysterol binding proteins on tyrosine phosphorylation of sperm proteins. The hyperactivated motility pattern of capacitated sperm relates to an increase in tyrosine phosphorylation of proteins in the flagellum [27] and we examined whether tyrosine phosphorylation occurred in incubated sperm samples. When sperm were incubated in bicarbonate enriched media (+Bic-BSA) only a weak protein tyrosine phosphorylation increase as compared to control sperm was recovered (1.1 fold; Fig. 5C). Inclusion of albumin (+Bic+BSA) caused an almost 4-fold increase in protein tyrosine phosphorylation (Fig. 5C). The $4 \mu \mathrm{g} / \mathrm{ml}$ dose of ORP-1 or ORP-2 (eliciting maximal stimulation in hyper-activated sperm motility; see Fig. 5B) caused a significant (approx. 2 fold) but less prominent induction of protein tyrosine phosphorylation compared to control sperm (Fig. 5C). Interestingly, when compared to albumin both ORPs induced tyrosine phosphorylation in the sperm head of a larger subpopulation of sperm cells (Fig. 5D) and this was manifested at the apical ridge area and at the equatorial area of the sperm head (Fig. 5D). Induction of tyrosine phosphorylation was not 
observed when vitamin E or A was added to bicarbonate enriched media either in presence or absence albumin or albumin (data not shown) for mouse sperm see Table 3.

Effects of oxysterol binding proteins on aggregation of flotillin into the apical ridge area In a previous study the depletion of sterols and aggregation of lipid raft marker proteins such as flotillin has been demonstrated to result in capacitation of porcine sperm [2]. We now have extended this observation and studied whether, as compared to sperm treated in bicarbonate enriched media, the albumin effect on sterol extraction and lipid raft aggregation could be mimicked by incubating sperm in bicarbonate enriched media supplemented with recombinant ORP-1 or -2. Clearly, as reported previously [2] and by others [12], inclusion of BSA to the bicarbonate enriched medium ( $+\mathrm{Bic}$ ) caused the aggregation of flotillin at the apical ridge area (for topology see insert of Fig. 6). In absence of (oxy)sterol depleting agents only $<20 \%$ of the sperm cells had this flotillin distribution and this proportion increased to $70 \%$ in presence of BSA (Fig. 6). When sperm were incubated in bicarbonate enriched media with either ORP-1 or ORP-2 this also resulted in a significant increase of aggregation of flotillin in the apical area of the sperm head although $4 \mu \mathrm{g} / \mathrm{ml}$ ORP-2 was much more efficient (to a similar degree as BSA) when compared to $4 \mu \mathrm{g} / \mathrm{ml}$ ORP-1 (Fig. 6). This phenomenon was not observed when vitamin E or vitamin A was added to bicarbonate enriched media either in presence or absence albumin (data not shown).

Effects of oxysterol binding proteins and antioxidants on the generation of zona affinity of sperm and in vitro fertilization

In vitro capacitation leads to the generation of hyper-activated sperm motility but also to the generation of affinity for the zona pellucida. In absence of bicarbonate (-Bic) no sperm bound to the zona pellucida (data not shown). Only a minimal amount of sperm cells showed binding to the zona pellucida ( 0.8 sperm cells per zona pellucida on average) when incubated in bicarbonate enriched-medium in the absence of (oxy)sterol interacting proteins (-BSA; Fig. 7A). When incubated in presence of BSA an average of 8 sperm cells were binding to each zona pellucida (+BSA Fig. 7A). Incubation of sperm in bicarbonate enriched media (+Bic) with ORP-1 also induced sperm zona binding (2-3 sperm cells per zona pellucida) whereas ORP-2 failed to induce this compared to bicarbonate-enriched medium without (oxy)sterol interacting proteins (Fig. 7A). The fertilization rates of sperm cells incubated under these four conditions were also followed. In vitro fertilization in the presence of bicarbonate and albumin (+BSA) led to a fertilization rate of $60 \%$ (Fig. 7B) where omission of (oxy)sterol binding proteins (-BSA) only led to $2.4 \%$ fertilization rates. Despite of the positive effects of ORPs on signs of sperm capacitation they led to only marginal increases of fertilization rates to $3.5 \%$ and $4.1 \%$ for a 4 $\mu \mathrm{g} / \mathrm{ml}$ dose of ORP-1 and ORP-2, respectively. In Fig. 7C we depicted the localization and presence of sperm when interacting with the oocyte-zona complex under IVF conditions. In the absence of (oxy)sterol interacting proteins almost no interacting sperm cells were detected. In the presence of ORP-1 or -2 some sperm cells were present at the zona pellucida and showed some signs of zona penetration indicating that these cells not only interact with the zona pellucida but also had induced the acrosome reaction which is a step required for zona penetration (Fig. 7C). However, a deeper penetration as shown for BSA was only rarely found under ORP-1 or ORP-2 incubations. 
Effects of oxysterol binding proteins on the induction of the acrosome reaction

The observation that fertilization rates were low in the presence of ORPs in bicarbonate-enriched media (in contrast to BSA) but that sperm incubated with ORPs showed some zona binding affinity and some zona penetration ability, led us to investigate whether ORPs were inducing premature acrosome reactions. Incubation of sperm in bicarbonate depleted medium or in bicarbonate-enriched medium (either in absence or presence of BSA) did not induce the acrosome reaction (the amount of acrosome reacted cells that stained positive for fluorescent conjugated peanut agglutinin; PNA-FITC, positive cells was approx. 10\%; Fig. 8). Note that BSA had a stabilizing effect on the acrosome integrity, which has been explained in a previous study showing that this condition causes stabilization of docked SNARE complexes [18]. In contrast, ORP- 1 and ORP-2 caused premature acrosome reactions in $20-30 \%$ of the sperm cells (Fig. 8B and C).

\section{Albumin, oxysterol binding proteins and methyl $\beta$-cyclodextrin efficiently reduce oxysterols levels in bicarbonate stimulated sperm.}

Experiments were designed in which sperm cells were treated in presence of bicarbonate $(+\mathrm{Bic})$ in absence and in presence of agents that can reduce sterols and/or oxysterols in sperm. To this end we used albumin, MBCD, or the recombinant oxysterol binding proteins ORP1 and ORP2. All agents caused a reduction of $50 \%$ or more of the oxysterols formed, when compared to the amount of oxysterols formed under bicarbonate alone (Fig 9A). Interestingly, both ORPs at 4 $\mu \mathrm{g} / \mathrm{ml}$ caused a very reproducible and more pronounced reduction in oxysterols levels than albumin or MBCD (a reduction of 70\%; Fig 9A).

\section{Desmosterol and cholesterol are depleted by albumin but not by oxysterol binding proteins in bicarbonate stimulated sperm}

Sperm stimulated with bicarbonate in the presence of albumin (+Bic+BSA) showed a $63 \%$ decrease of cholesterol and almost $34 \%$ decrease in desmosterol when compared to the negative control (no bicarbonate) (Supplemental Figure S1, available online at www.biolreprod.org) which was recovered in the albumin containing supernatant after centrifugation (Fig. 8B, Table 2). The depletion of these sterols could be inhibited by vitamin $\mathrm{E}$ or vitamin A (Fig. 8B). Independent of bicarbonate inclusion the MBCD also caused a significant (but lower) depletion of cholesterol (27\% and $19 \%)$ and desmosterol (27\% and 33\%) in absence and presence of bicarbonate respectively in line with previous studies (Fig. 9B and Supplementary Data [2, 3]). Desmosterol is a precursor sterol in the cholesterol biosynthetic pathway present in all samples in a molar ratio of $1.5 \%$ relative to cholesterol. Note that the intensity of ions detected as expressed in Fig. 2 are detected with varying levels of sensitivity due to the different ionization efficiencies in the APCI process. They were corrected for a response curve made for each individual oxysterol species and sterol species detected as described previously [4]. Interestingly, the addition of pro-oxidants in presence of albumin and bicarbonate (i.e. under oxysterol forming conditions, see Fig. 1) did only result in modest reduce the level of cholesterol (13\%) but a pronounced reduction of desmosterol ( $43 \%$ ) when compared to the -Bic+BSA control (Fig. 9B; Supplemental Figure S1). Moreover, the two oxysterol binding proteins ORP-1 and ORP-2, although very efficient in reducing the oxysterols levels in sperm cells (see Fig. 9A), did not cause significant reductions in levels of cholesterol or desmosterol (Fig. 9B). The incubations with bicarbonate and BSA caused the depletion of oxysterols cholesterol and desmosterol from 
the sperm surface the medium (for partition of each sterol component in in vitro capacitated mouse and boar sperm (see Tables 1 and 2).

$M B C D$ mediated sterol depletion causes tyrosine phosphorylation and hyper-activated motility in a different fashion than recombinant ORPs or BSA

In addition to the effects of recombinant ORP-1 and -2 on sperm capacitation we compared the effects of a much less specific sterol depletor on sperm capacitation, MBCD. In Fig. 9 it was already shown that MBCD has the capacity to deplete the bicarbonate dependent production of oxysterols. However, MBCD is a potent depletor of free sterols both in the absence and the presence of bicarbonate (Fig. 9 and [28-30]). In fact in our hands in the dose range of $0.5-10 \mathrm{mM}$ MBCD caused an increase in tyrosine phosphorylation both in the absence (-Bic) and the presence $(+\mathrm{Bic})$ of bicarbonate (Fig. 10A), with highest stimulation to similar levels as that of BSA (see Fig. 4C; note that BSA did only have this effect in the presence of bicarbonate). In the absence of bicarbonate only the specific concentration of $2 \mathrm{mM} \mathrm{MBCD}$ led to the induction of hyper-activated sperm motility (Fig. 10B) whereas in presence of bicarbonate MBCD did not attenuate the bicarbonate induction of hyper-activated sperm motility and significantly inhibited this response at higher dosages (Fig. 9B).

$M B C D$ treatment induce low in vitro fertilization rates and oocyte degeneration In analogy of the IVF experiments in bicarbonate enriched media with BSA or with recombinant ORPs, we tested the effect of MBCD on fertilization rates. The fertilization rates after treatment of sperm with 0.5-10 mM MBCD compared to of BSA (for BSA $>60 \%$ see Fig. 7B) were dramatically reduced (Fig. 10C). However, in contrast to ORP-1 and 2 incubations the $2 \mathrm{mM}$ MBCD condition led to much lower fertilization rates $(30 \%)$. At lower and higher levels of MBCD ( 0.5 and $10 \mathrm{mM}$ respectively) even lower fertilization rates were observed when compared to sperm treated with 0mM MBCD; Fig. 10C, or with recombinant ORPs (cf. Fig. 7B). A dose-dependent degeneration of oocytes was observed when IVF was performed in the presence $0.5-10 \mathrm{mM}$ MBCD. In the presence of BSA these rates were $<10 \%$ (Fig. 7B) whereas 2 and $10 \mathrm{mM}$ MBCD caused unacceptably high levels of oocyte degeneration (of 30 and $100 \%$ respectively Fig. 10C).

\section{DISCUSSION}

\section{Induction and prevention of oxysterol formation}

In this manuscript the formation of oxysterols is described in sperm under in vitro capacitating conditions. The oxysterol formation was dependent on bicarbonate ions that must have induced the formation radical oxygen species [31] because oxysterol formation was essentially blocked when mouse or porcine sperm cells were treated in bicarbonate enriched media in the presence of vitamin $\mathrm{E}$ or vitamin $\mathrm{A}$. In a previous manuscript we have shown from bovine sperm that oxysterol formation can be induced by incubating sperm in presence of pro-oxidants like tertbutylhydroxide [4]. Here we showed that oxysterols can be formed in the absence of bicarbonate under certain pro-oxidant conditions $\left(\mathrm{FeSO}_{4}+\right.$ ascorbate) but not by the peroxynitrite forming agent SIN-1. Both bicarbonate and the $\mathrm{FeSO}_{4}+$ ascorbate incubations induced especially the production of 7-ketocholesterol and 5,6 $\beta$-epoxycholesterol.

Oxysterol formation and interaction with oxysterol binding proteins induces various characteristics of sperm capacitation 
When sperm are capacitated in vitro in the presence of bicarbonate and BSA they show signs of hyper-activated sperm motility [27], increased levels of tyrosine phosphorylation [27], surface changes (including the already noted removal of sterols [32]), redistribution and aggregation of lipid microdomains [12], and higher affinity for the zona pellucida [11, 16]. These changes together result in the sperm becoming competent to fertilize the oocyte during IVF incubations [32]. In the present work, in which BSA was replaced for either recombinant ORP-1 or ORP-2, indeed a number of capacitation-responses including (i) the induction of hyper-activated sperm motility; (ii) increased tyrosine phosphorylation; (iii) aggregation of lipid-ordered microdomain membrane markers at the apical ridge area of the sperm head; and (iv) to some extend higher affinity for the zona pellucida, were detected. In fact, ORP treatment led to some limited penetration activity of sperm cells through the zona pellucida but this response was limited when compared to BSA. All these responses were only present when sperm were incubated in bicarbonate-rich media (+Bic) and the responses were inhibited in presence of vitamin $\mathrm{E}$ or blocked by vitamin A. Thus an essential part of sperm capacitation is due to the formation of oxysterols and putatively to the interaction of these formed oxysterols with proteins involved in sterol depletion at the sperm surface $[8,19]$.

\section{Depletion of oxysterols, desmosterol, and cholesterol}

When sperm capacitation was performed in presence of the sterol-depleting molecule BSA (routinely done in IVF [11]) this resulted in an efficient reduction of the majority of oxysterols $(50 \%)$ from the sperm surface into the albumin containing fraction not associated to the sperm surface (recovered in the supernatant after centrifugation). An even more severe reduction of oxysterols were detected when sperm were incubated with recombinant oxysterol binding proteins (ORP-1 and ORP-2; in both cases a reduction of $>70 \%$ of the oxysterols took place), which are known to bind oxysterols $[11,33]$. Importantly, ORPs are soluble proteins enhancing exchange of sterols from donor to acceptor membranes over the cytosol in a variety of cells. After isolation of these two recombinant proteins from E.coli we have used them as tools for the specific interaction of oxysterols in sperm suspensions under various in vitro capacitation conditions. We must stress here that this extracellular type of action is not mimicking eventually endogenous ORPs under physiological conditions where they (if at all present in sperm) are active intracellularly. The effects of ORPs, similar to BSA, were only seen in bicarbonateenriched media but not in the absence of bicarbonate. When sperm were incubated with Bic + ORPs no depletion of cholesterol or desmosterol was monitored while Bic + BSA caused a depletion of $>30 \%$ cholesterol and desmosterol. Interestingly, ORP treatment only caused a minor $(<10 \%)$ of the oxysterols in the supernatant after centrifugating sperm. This implies that ORP (in contrast to BSA) does not deplete oxysterols efficiently from sperm but scavenges oxysterols (by binding to the sperm surface?) during the induction of their production at the sperm surface by bicarbonate. Probably, the bicarbonate-induced capacitation responses are not inhibited (instead it was even promoted) by ORP treatment as the intracellular ROS formation and ROS signaling is not affected. It seems that the concomitant high levels of oxysterols in sperm treated with bicarbonate alone, can be compensated by albumin (depletion) or by ORP (likely surface binding and scavenging). Moreover, oxysterol formation was inhibited by pretreating sperm with vitamin A this caused a complete block of in vitro fertilization and while vitamin $\mathrm{E}$ had only a partial inhibiting effect. Both membrane antioxidants also inhibited sperm zona binding but not the integrity of sperm or oocytes. These findings indicate that lowering ROS and ROS dependent signaling processes at the sperm surface prior to an in vitro fertilization 
experiment blocks oxysterol formation, sperm capacitation and reduces sperm-zona binding and IVF rates. The difference in degree of effect between vitamin A and vitamin $\mathrm{E}$ can lie in intrinsic properties of both hydrophobic antioxidants and the way we pretreated sperm prior to IVF. It is possible that vitamin A either resides better in the sperm surface during the IVF procedure (more hydrophobic) or elicits its antioxidant properties more efficiently when compared to vitamin $\mathrm{E}$ (vitamin A is a stronger antioxidant). Note that the pretreated sperm was diluted 10 times and used for 24 hours in an IVF experiment. Under such conditions back exchange of the antioxidants to albumin may occur. The decrease of IVF rates by vitamin $\mathrm{E}$ has been reported for bovine sperm [34,35]. Moreover, inseminating oocytes with hydrogen peroxide pretreated sperm has been shown to increase fertilization, cleavage and blastocyst rates [36]. Taken together we believe that these data show that depletion of sperm surface lipid peroxides (or in absence of bicarbonate the lack of formation of sperm surface lipid peroxides) cause a reduction in IVF rates. The formation of lipid peroxides under bicarbonate allows reverse transport of (oxy)sterols to BSA and high IVF rates while excessive lipid peroxidation is detrimental to the sperm cell and thus for fertilization [10].

\section{Oxysterol reduction alone is insufficient for obtaining IVF success}

Interestingly, unlike albumin, the ORPs were not able to reduce cholesterol or desmosterol, which fits to their higher specific affinity for oxysterols when compared to albumin. Probably this lack of reducing levels of free sterols at the sperm surface relates to the fact that incubations of BSA did result in $60 \%$ fertilization rates after IVF whereas ORP incubations largely failed to result in fertilization. ORP as well as BSA incubations both induced in vitro sperm capacitation although ORP also induced preliminary acrosome reactions while BSA treated cells remained acrosome intact. Therefore, it is possible that the concentration and length of ORP treatments need to be refined and that the currently used circumstances were simply too rough for the sperm to become fertilization-competent. A support for this possibility is that the lowest dose of ORP-1 or ORP-2 caused already maximal stimulation of hyper-activated motility and higher doses were partly inhibiting this effect. The above mentioned possibility that ORPs act to inhibit oxysterol formation by scavenging oxysterols may at higher doses become a too efficient process and thus will inhibit ROS dependent capacitation responses. Presently we are studying how extracellular added ORPs on the sperm surface cause the cellular responses that in part run parallel to bicarbonate/BSA induced capacitation. The oxysterol binding protein family contains $>10$ proteins and their role in cell physiology has recently been reviewed [37]. Of course another possibility for the discrepancies between BSA and ORPs may lie in that BSA has other (oxysterol-independent) effects on sperm [38].

\section{$M B C D$ causes an -in part- bicarbonate-independent sterol depletion from sperm}

Besides BSA and ORP, another molecule with affinity for sterols, MBCD, has been exploited either to elicit sperm capacitation [30] or to achieve BSA independent in vitro fertilization [39]. The latter can be useful in developing methods to treat sperm under fully pathogen free declared materials. Because only a part of the BSA effects can be obtained by ORP replacement the effects of MBCD were tested. As reported in the literature MBCD is in our hands indeed capable of inducing hyper-activated sperm motility $[40,41]$ and tyrosine phosphorylation in the sperm tail [27, 40], and causes a depletion of cholesterol, desmosterol [28] and oxysterols (this study). However, MBCD does work in a different fashion when compared to BSA and ORP1/2 as it elicits these effects independently from bicarbonate and does not stop extracting sterols in 
presence of vitamin E [28]; this study. Moreover, MBCD fails to induce aggregation of flotillin and tyrosin phosphorylation in the sperm head [28] and extracts sterols from both lipid rafts and from the non-raft membranes whereas BSA only extracts sterols from the non-raft domain [2]. In the presence of bicarbonate higher levels of MBCD inhibit sperm motility (this study) and also cause sperm membrane deterioration and premature acrosome reactions [29]. Despite of all these differences to BSA, $2 \mathrm{mM}$ MBCD incubation led to in vitro fertilization rates of $30 \%$. However, already at this concentration MBCD caused a non-acceptable level of oocyte degeneration $(>30 \%)$ which was observed for all oocytes at $10 \mathrm{mM}$ MBCD.

In conclusion, the bicarbonate-dependent formation of reactive oxygen species leads to the production of oxysterols. Probably, the subsequent interactions of the formed oxysterols with oxysterol-binding proteins (ORPs or albumin) and substantial depletion of oxysterols enable sperm capacitation and related specific sperm surface changes [13]. We postulate that that these subtle changes in sperm sterols (only less than $1 \%$ of sterols are converted to oxysterols) may activate a sterol transporter protein. In this respect it is well possible that a protein like the CD36 (a multifunctional protein homologous to the class B scavenger receptor SR-B1) acts as a lipid sensor [42] to oxysterols. In fact a recent report on bull sperm has indicated that CD36 is a biomarker for male fertility [43]. SR-B1 itself (translation product from the $S A R B-1$ gene) has also been implied in regulating reverse cholesterol transport to high density lipoproteins in other tissues [44] and could be activated by surface rearrangements typical for capacitating sperm as postulated previously [1]. Other candidates for a reverse sterol transporter potentially active at the sperm surface belong to the ATP cassette transporters; ABCA1,-7,-17 and ABCG1 are all detected in sperm $[45,46]$, and both $\mathrm{ABCA} 1$ and $\mathrm{ABG} 1$ have in fact been shown to transport oxysterols out of cells [9].

Our results provide evidence that only with a cholesterol/desmosterol accepting protein in the sperm incubation buffer, sperm became capable of fertilizing the oocyte. ORP-1 and 2 turned out to be too specific for reducing oxysterol levels but failing to deplete cholesterol/desmosterol, which could render the treated sperm unfertile under IVF conditions. The sperm surface interaction of ORP also induced some premature acrosome reactions. BSA was under bicarbonate conditions well capable of extracting cholesterol desmosterol and oxysterols, thus allowing depletion of a large amount of total free sterols, an effect apparently required for fertilization. Blocking oxysterol formation in the sperm membrane with hydrophobic antioxidants reduced sperm motility tail tyrosine phosphorylation and IVF rates and to a lesser extend inhibited sperm-zona binding. This may indicate that ROS formation and peroxidation of sterols (and possibly other lipids) in the sperm membrane is required for proper zona penetration rather than the recognition of this structure. The bicarbonate and ROS independent sterol depletion by MBCD also induces some aspects of sperm capacitation but fails to generate good IVF results due to adverse effects on oocyte physiology. Probably, more subtle treatments with ORPs or MBCD could result in better IVF results. The balance between the rate of oxysterol formation and of oxysterol depletion are probably determining how the sperm are going through the time-window of sperm capacitation before to reach the state of deterioration (see model in Figure 11 and [31]). Thus, this study has shed new light on the mechanisms of how oxysterols are formed in the sperm and that this process precedes free sterol depletion from the sperm surface during in vitro fertilization. Apparently a bicarbonate induced ROS dependent membrane process is required to activated reverse (oxy)sterol transport from the capacitating sperm surface. 
From this study it is not clear whether the formation of oxysterols itself is involved in the activation of a reverse sterol transporter or whether they are just molecular signs of ROS emerging in the sperm surface and indirectly linked to the depletion of (oxy)sterols by albumin. Future research should focus on the exact conditions that can be used to optimize in vitro fertilization with sterol depleting agents. More importantly experiments should be planned to unravel the mechanism of reverse sterol transport from capacitating sperm as well as determining the involvement of oxysterols in this sterol transport which is crucial for in vitro fertilization.

\section{ACKNOWLEDGEMENTS}

Dr. R.L. Serrano has helped with the transfection experiments and purification of recombinant ORP-1 and ORP-2 proteins. Dr. P.S. Tsai has helped with IVF and sperm incubation experiments.

\section{REFERENCES}

1. Flesch FM, Gadella BM. Dynamics of the mammalian sperm plasma membrane in the process of fertilization. Biochim Biophys Acta 2000; 1469:197-235.

2. van Gestel RA, Brewis IA, Ashton PR, Helms JB, Brouwers JF, Gadella BM. Capacitationdependent concentration of lipid rafts in the apical ridge head area of porcine sperm cells. Mol Hum Reprod 2005; 11:583-590.

3. Flesch FM, Brouwers JF, Nievelstein PF, Verkleij AJ, van Golde LM, Colenbrander B, Gadella BM. Bicarbonate stimulated phospholipid scrambling induces cholesterol redistribution and enables cholesterol depletion in the sperm plasma membrane. J Cell Sci 2001; 114:35433555 .

4. Brouwers JF, Boerke A, Silva PF, Garcia-Gil N, van Gestel RA, Helms JB, van de Lest CH, Gadella BM. Mass Spectrometric Detection of Cholesterol Oxidation in Bovine Sperm. Biol Reprod 2011; 85:128-136.

5. Boerke A, Tsai PS, Garcia-Gil N, Brewis IA, Gadella BM. Capacitation-dependent reorganization of microdomains in the apical sperm head plasma membrane: Functional relationship with zona binding and the zona-induced acrosome reaction. Theriogenology 2008; 70:1188-1196.

6. de Lamirande E, Lamothe G, Villemure M. Control of superoxide and nitric oxide formation during human sperm capacitation. Free Radic Biol Med 2009; 46:1420-1427.

7. de Lamirande E, Lamothe G. Reactive oxygen-induced reactive oxygen formation during human sperm capacitation. Free Radic Biol Med 2009; 46:502-510.

8. van der Vusse GJ. Albumin as fatty acid transporter. Drug Metab Pharmacokinet 2009; 24:300-307.

9. Jessup W, Gelissen IC, Gaus K, Kritharides L. Roles of ATP binding cassette transporters A1 and G1, scavenger receptor BI and membrane lipid domains in cholesterol export from macrophages. Curr Opin Lipidol 2006; 17:247-257.

10. Aitken RJ, Baker MA. Oxidative stress, sperm survival and fertility control. Mol Cell Endocrinol 2006; 250:66-69.

11. Blake D, Svalander P, Jin M, Silversand C, Hamberger L. Protein supplementation of human IVF culture media. J Assist Reprod Genet 2002; 19:137-143.

12. Zitranski N, Borth H, Ackermann F, Meyer D, Viewig L, Breit A, Gudermann T, Boekhoff I. The "acrosomal synapse": Subcellular organization by lipid rafts and scaffolding proteins 
exhibits high similarities in neurons and mammalian spermatozoa. Commun Integr Biol 2010; 3:513-521.

13. Thaler CD, Thomas M, Ramalie JR. Reorganization of mouse sperm lipid rafts by capacitation. Mol Reprod Dev 2006; 73:1541-1549.

14. Gadella BM, Tsai PS, Boerke A, Brewis IA. Sperm head membrane reorganisation during capacitation. Int J Dev Biol 2008; 52:473-480.

15. Nixon B, Aitken RJ. The biological significance of detergent-resistant membranes in spermatozoa. J Reprod Immunol 2009; 83:8-13.

16. van Gestel RA, Brewis IA, Ashton PR, Brouwers JF, Gadella BM. Multiple proteins present in purified porcine sperm apical plasma membranes interact with the zona pellucida of the oocyte. Mol Hum Reprod 2007; 13:445-454.

17. Tsai P, De Vries KJ, De Boer-Brouwer M, Garcia-Gil N, Van Gestel RA, Colenbrander B, Gadella BM, Van Haeften T. Syntaxin and VAMP association with lipid rafts depends on cholesterol depletion in capacitating sperm cells. Mol Membr Biol 2007; 24:313-324.

18. Tsai PS, Garcia-Gil N, van Haeften T, Gadella BM. How pig sperm prepares to fertilize: stable acrosome docking to the plasma membrane. PLoS One 2010; 5:e11204.

19. Tsai PS, Gadella BM. Molecular kinetics of proteins at the surface of porcine sperm before and during fertilization. Soc Reprod Fertil Suppl 2009; 66:23-36.

20. Aitken RJ, Clarkson JS, Fishel S. Generation of reactive oxygen species, lipid peroxidation, and human sperm function. Biol Reprod 1989; 41:183-197.

21. Suchanek M, Hynynen R, Wohlfahrt G, Lehto M, Johansson M, Saarinen H, Radzikowska A, Thiele C, Olkkonen VM. The mammalian oxysterol-binding protein-related proteins (ORPs) bind 25-hydroxycholesterol in an evolutionarily conserved pocket. Biochem J 2007; 405:473480.

22. Wilson CM. Staining of proteins on gels: comparisons of dyes and procedures. Methods Enzymol 1983; 91:236-247.

23. Gergel D, Misik V, Ondrias K, Cederbaum AI. Increased cytotoxicity of 3morpholinosydnonimine to HepG2 cells in the presence of superoxide dismutase. Role of hydrogen peroxide and iron. J Biol Chem 1995; 270:20922-20929.

24. Broekhuijse ML, Sostaric E, Feitsma H, Gadella BM. Additional value of computer assisted semen analysis (CASA) compared to conventional motility assessments in pig artificial insemination. Theriogenology 2011; 76:1473-86.e1.

25. Schoevers EJ, Kidson A, Verheijden JH, Bevers MM. Effect of follicle-stimulating hormone on nuclear and cytoplasmic maturation of sow oocytes in vitro. Theriogenology 2003; 59:2017-2028.

26. Harrison RA. Capacitation mechanisms, and the role of capacitation as seen in eutherian mammals. Reprod Fertil Dev 1996; 8:581-594.

27. Bajpai M, Asin S, Doncel GF. Effect of tyrosine kinase inhibitors on tyrosine phosphorylation and motility parameters in human sperm. Arch Androl 2003; 49:229-246.

28. van Gestel RA, Helms JB, Brouwers JF, Gadella BM. Effects of methyl- $\beta$-cyclodextrinmediated cholesterol depletion in porcine sperm compared to somatic cells. Mol Reprod Dev 2005; 72:386-395.

29. Shadan S, James PS, Howes EA, Jones R. Cholesterol Efflux Alters Lipid Raft Stability and Distribution During Capacitation of Boar Spermatozoa. Biology of Reproduction 2004; 71:253-265. 
30. Choi YH, Toyoda Y. Cyclodextrin removes cholesterol from mouse sperm and induces capacitation in a protein-free medium. Biol Reprod 1998; 59:1328-1333.

31. Aitken RJ. The Capacitation-Apoptosis Highway: Oxysterols and Mammalian Sperm Function. Biol Reprod 2011; 85:9-12.

32. Osheroff JE, Visconti PE, Valenzuela JP, Travis AJ, Alvarez J, Kopf GS. Regulation of human sperm capacitation by a cholesterol efflux-stimulated signal transduction pathway leading to protein kinase A-mediated up-regulation of protein tyrosine phosphorylation. Mol Hum Reprod 1999; 5:1017-1026.

33. Yan D, Olkkonen VM. Characteristics of oxysterol binding proteins. Int Rev Cytol 2008; 265:253-285.

34. Marques A, Santos P, Antunes G, Chaviero P, Moriero da Silva F. Effect of $\alpha$-tocopherol on bovine in vitro fertilization. Reprod Dom Anim 2010; 45:81-85.

35. Dalvit GC, Cetica PD, Beconi MT. Effect of $\alpha$-tocopherol and ascorbic acid on bovine in vitro fertilization. Theriogenology 1998; 49:619-627.

36. Rahman MB, Vandaele L, Rijsselaere T, Zandi M, Maes D, Shamsuddin M, van Soom A. Oocyte quality determines bovine embryo development after fertilization with hydrogen peroxide-stressed spermatozoa. Reprod Fert Dev 2012; 24:608-618.

37. Vihervaara T, Jansen M, Uronen RL, Ohsaki Y, Ikonen E, Olkkonen VM. Cytoplasmic oxysterol-binding proteins: sterol sensors or transporters? Chem Phys Lipids 2011; 164:443-450. 38. Xia J, Ren D. The BSA-induced $\mathrm{Ca}(2+)$ influx during sperm capacitation is CATSPER channel-dependent. Reproductive Biology and Endocrinology 2009; 7:119.

39. Takeo T, Hoshii T, Kondo Y, Toyodome H, Arima H, Yamamura K, Irie T, Nakagata N. Methyl-beta-cyclodextrin improves fertilizing ability of C57BL/6 mouse sperm after freezing and thawing by facilitating cholesterol efflux from the cells. Biol Reprod 2008; 78:546-551.

40. Kato Y, Shoei S, Nagao Y. Capacitation status of activated bovine sperm cultured in media containing methyl-beta-cyclodextrin affects the acrosome reaction and fertility. Zygote 2011; 19:21-30.

41. Visconti PE, Galantino-Homer H, Ning X, Moore GD, Valenzuela JP, Jorgez CJ, Alvarez JG, Kopf GS. Cholesterol efflux-mediated signal transduction in mammalian sperm. betacyclodextrins initiate transmembrane signaling leading to an increase in protein tyrosine phosphorylation and capacitation. J Biol Chem 1999; 274:3235-3242.

42. Martin C, Chevrot M, Poirier H, Passilly-Degrace P, Niot I, Besnard P. CD36 as a lipid sensor. Physiol Behav 2011; 105:36-42.

43. Feugang JM, Rodriguez-Osorio N, Kaya A, Wang H, Page G, Ostermeier GC, Topper EK, Memili E. Transcriptome analysis of bull spermatozoa: implications for male fertility. Reprod Biomed Online 2010; 21:312-324.

44. Nieland TJ, Chroni A, Fitzgerald ML, Maliga Z, Zannis VI, Kirchhausen T, Krieger M. Cross-inhibition of SR-BI- and ABCA1-mediated cholesterol transport by the small molecules BLT-4 and glyburide. J Lipid Res 2004; 45:1256-1265.

45. Morales CR, Marat AL, Ni X, Yu Y, Oko R, Smith BT, Argraves WS. ATP-binding cassette transporters ABCA1, ABCA7, and ABCG1 in mouse spermatozoa. Biochem Biophys Res Commun 2008; 376:472-477.

46. Ban N, Sasaki M, Sakai H, Ueda K, Inagaki N. Cloning of ABCA17, a novel rodent spermspecific ABC (ATP-binding cassette) transporter that regulates intracellular lipid metabolism. Biochem J 2005; 389:577-585. 


\section{Figure legends}

Fig. 1 Oxysterol formation is induced by a bicarbonate-activated reactive oxygen species dependent pathway. Bicarbonate enriched medium (+Bic) induces radical formation, leads to oxidation of cholesterol and can be blocked by vitamin $\mathrm{E}$ or by vitamin A. Oxysterols were detected by mass spectrometry see [20]. The relative amount of oxysterols as percentage of cellular cholesterol is expressed (mean $\% \pm$ SEM, $n=3$ ejaculates were obtained from individual boars). * indicate a significant induction of oxysterol formation when compared to $-\mathrm{Bic}(\mathrm{P}<0.05)$

Fig. 2 Chromatograms of multiple reaction monitoring during the elution of (oxy-)sterols extracted from sperm cells. Oxysterols were identified based on retention time and product spectra as annotated: Triol, 3,5,6-trihydroxycholesterol; 7-OH, 7-hydroxycholesterol; 7-keto, 7ketocholesterol; 22-keto, 22-ketocholesterol; 25-OH, 25-hydroxycholesterol; $\beta$-epoxy, 5,6 $\beta$ epoxycholesterol; $\alpha$-epoxy, 5,6 $\alpha$ - epoxycholesterol. Each panel represents oxysterols from one million sperm cells. The amount of desmosterol and cholesterol (note the discontinuous Y-axis to accommodate visualization of the peak height of these much more abundant sterol species) is depicted as well. The elution profile with $m z$ transitions intensities of 367.2/159.1 were used for oxysterols and desmosterol (indicated as 367) the transition of 369.2/161.1 for cholesterol (indicated as 369) and the transition of 401.2/175.1 for ketosterols (indicated as 401).

Fig. 3 Relative molar contributions of cellular oxysterol species to the total oxysterol content in sperm cells after incubation in media supplemented and centrifugation as indicated. Note the relative decrease of 7-OH and increase of 7-ketocholesterol under conditions with increased oxysterol formation. The top panels refer to $-\mathrm{Bic}$ and + Bic were both in absence of albumin. Conditions marked ' $a$.' where control levels of oxysterol formation were observed. Conditions marked ' $b$.' where oxysterol levels were approx. 10-fold higher than under control conditions (data in Fig. 1). For the definitions of the oxysterol species abbreviations used see Fig. 2 (Numbers in graph depict sum of oxysterol species: mean \pm SD in pmol/10 cells; $n=3$ ).

Fig. 4 Effects of the bicarbonate/ROS pathway on hyperactivated motility, zona binding and in vitro fertilization. A) Vitamin $\mathrm{E}$ and vitamin $\mathrm{A}$ inhibit the bicarbonate and albumin induced of hyper-activated sperm motility. Bicarbonate in absence (+Bic-BSA) and especially in presence of albumin (+Bic+BSA) induces hyper-activated sperm motility. In presence of vitamin $\mathrm{E}$ or vitamin A no signs of hyper-activated sperm motility where seen in absence of albumin (+Bic$\mathrm{BSA}+\mathrm{VitE})$ and in presence of albumin $(+\mathrm{Bic}+\mathrm{BSA}+\mathrm{VitE} ;+\mathrm{Bic}+\mathrm{BSA}+\mathrm{VitA})$ the levels of hyper-activated sperm motility were comparable to control sperm that were incubated in absence of bicarbonate and albumin (-Bic-BSA). The data represent mean $\pm \mathrm{SEM}, \mathrm{n}=3$. * indicate a significant increase of effects compared to-Bic-BSA; *** indicate a significant lower effect compared to $+\mathrm{Bic}+\mathrm{BSA}$ and a significant effect compared to -Bic-BSA; \# indicates a significant lower amount of hyperactive motile sperm when compared to-Bic-BSA (in all predictions $\mathrm{P}<0.05)$. B) Sperm binding to oocytes after a pretreatment of 30 minutes in the IVF medium containing $0.1 \%$ ethanol (control) or in $0.5 \mathrm{mM}$ of vitamin A (VitA) or vitamin $\mathrm{E}$ (VitE). The individual black squares indicate the amount of sperm that bound to the zona pellucida for each of the boar ejaculates tested ( 8 different boars indicated A-H on the x-axis). The mean amount of sperm bound to unfertilized oocytes (M2) monospermic fertilized oocytes (Mono) and polyspermic fertilized oocytes (Poly) is indicated in the top panels. For control and VitE 8 individual boars were scored for VitA 3 boars were scored and no fertilized oocytes were 
detected. C) Vitamin A blocked in and vitamin E significantly reduced $(\mathrm{P}<0.05)$ IVF rates. The relative reduction compared to control IVF rates is expressed normalized fertilization (NFR). The fertilization rate (in $\% \mathrm{Y}$ axis) and the ratio of polyspermic fertilization/ total fertilization (in $\% \mathrm{X}$-axis) are indicated for ejaculated obtained from 11 individual AI boars. For each IVF treatment and each individual boar $>30$ oocytes were used.

Fig. 5. The effects of oxysterol binding proteins on sperm capacitation. B) Purification of recombinant oxysterol binding proteins ORP-1 and ORP-2. A) Silver stained SDS-PAGE cells loaded with ORP preparations: lane 1 protein supernatant isolated after ultracentrifugation of transfected $E$. coli suspensions, lane 2 supernatant after an additional bead treatment with glutathione sepharose, lane 3 the empty wash fraction of glutathione sepharose column containing and lane 4, the eluted proteins with $10 \mathrm{mM}$ glutathione which released the ORP-GSH fusion constructs (see also [21]). B) Inclusion of albumin (+Bic+BSA) and of 4-16 $\mu \mathrm{g} / \mathrm{ml} \mathrm{ORP-2}$ (+Bic+ORP-2) and for lower doses of 4 and to a lesser extent $8 \mu \mathrm{g} / \mathrm{ml} \mathrm{ORP}-1$ (+Bic+ORP-1) induced hyper-activated sperm motility. The highest dose of $16 \mu \mathrm{g} / \mathrm{ml} \mathrm{ORP}-1$ (+Bic+ORP-1) showed no increase of hyper-activated sperm motility and was comparable to the condition without added sterol depleting agents (+Bic-BSA). C) Western blot analysis revealed that tyrosine phosphorylation is increased 3.5 -fold in the bicarbonate and BSA condition $(+\mathrm{Bic}+\mathrm{BSA})$ when compared to the absence of sterol depleting agents (+Bic-BSA). Inclusion of $4 \mu \mathrm{g} / \mathrm{ml}$ ORP-1 (+Bic+ORP-1) or $4 \mu \mathrm{g} / \mathrm{ml}$ ORP-2 (+Bic+ORP-2) also induced tyrosine phosphorylation albeit to a smaller population of incubated sperm cells. D) Besides tail labeling (causing the hyper-activated motility), PY-20 labeling of tyrosine phosphorylated proteins was found on the apical ridge area (left arrow) and at the equatorial area (right arrow) of the sperm head. C) Clearly the inclusion of albumin (+Bic+BSA) $4 \mu \mathrm{g} / \mathrm{ml} \mathrm{ORP}-1$ (+Bic+ORP-1) or 4 $\mu \mathrm{g} / \mathrm{ml}$ ORP-2 (+Bic+ORP-2) to bicarbonate enriched media induced, in following increasing order, more PY20 labeling on both of the sperm head areas when compared to medium without sterol depleting agents (+Bic-BSA). The data represent mean $\pm \mathrm{SEM}, \mathrm{n}=3$. * indicate a significant increase of effects compared to $-\mathrm{Bic}-\mathrm{BSA} ; * *$ indicate a significant additional increase of effect when compared to $+\mathrm{Bic}+\mathrm{BSA}$; *** indicate a significant lower effect compared to $+\mathrm{Bic}+\mathrm{BSA}$ and a significant effect compared to -Bic-BSA; (in all predictions $\mathrm{P}<0.05)$

Fig. 6 The bicarbonate/oxysterol pathway induces protein tyrosine phosphorylation and aggregation of lipid ordered domains. Incubations with specific depletion of oxysterols result in aggregation of flotillin-1 into the apical ridge area of the sperm head. Incubation in presence of albumin (+Bic+BSA) or $4 \mu \mathrm{g} / \mathrm{ml}$ ORP2 (+BIC+ORP2) induced immunolabelling of flotillin-1 in the apical ridge area of the sperm head when compared to sperm cells incubated in absence of sterol depleting agents (+Bic-BSA). $4 \mu \mathrm{g} / \mathrm{ml}$ ORP-1 only (+Bic+ORP-1) slightly induced the apical ridge this relocation of flotillin-1. The data represent mean $\pm S E M, n=3$. * indicate a significant increase of effects compared to $+\mathrm{Bic}-\mathrm{BSA} ; * * *$ indicate a significantly lower effect compared to $+\mathrm{Bic}+\mathrm{BSA}$ and a significant effect compared to +Bic-BSA (in all comparisons $\mathrm{P}<0.05)$.

Fig. 7 The bicarbonate/oxysterol/BSA pathway generates zona affinity and in vitro fertilization but replacement of BSA by ORPs is insufficient. A) Sperm cells incubated in bicarbonate enriched medium with albumin (+Bic+BSA) showed affinity for the zona pellucida when 
compared to bicarbonate enriched medium without sterol depleting agents where essentially no zona binding was observed (+Bic-BSA). $4 \mu \mathrm{g} / \mathrm{ml}$ ORP-1 (+Bic+ORP-1) but not $4 \mu \mathrm{g} / \mathrm{ml} \mathrm{ORP-2}$ $(+\mathrm{Bic}+\mathrm{ORP}-2)$ caused a smaller but significant increase in zona affinity than $+\mathrm{Bic}+\mathrm{BSA}$ when compared to + Bic-BSA. B) Only the + Bic + BSA condition gave rise to efficient fertilization rates while + Bic+ORP-1 or + Bic+ORP-2 did not have positive effects when compared to +Bic-BSA. For each experiment and each incubation type at least 20 oocytes were used. The data represent mean \pm SEM, $n=3$. C) Representative microscopic photographs of sperm-zona interactions under the incubations provided in B. Green fluorescence indicated labeling of DNA and are depicted as merge with the corresponding bright field images obtained. The dashed lines indicate the border of the zona palluda. +Bic-BSA no sperm interaction with the zona pellucida was noted. In presence of albumin ( $+\mathrm{Bic}+\mathrm{BSA})$ full penetration through the zona pellucida was noted while interaction with the zona pellucida (+BSA+ORP-1) or slight penetration (+Bic+ORP-2) was noted when albumin was replaced with recombinant ORP.

Fig. 8 Sperm incubations with ORPs induce the acrosome reaction. A) Merged phase contrast image and PNA-FITC labeling of an acrosome intact sperm (left) and an acrosome reacted sperm cell (right). B) The relative amount of acrosome reacted sperm cells in absence (+Bic-BSA) and in presence of sterol depleting agents (+Bic+ORP-1; +Bic+ORP-2 or +Bic+BSA respectively). The doses of ORP was $4 \mu \mathrm{g} / \mathrm{ml}$. The data represent mean $\pm \mathrm{SEM}, \mathrm{n}=3$. Fluorescence intensity histograms of the PNA-FITC labeling of sperm incubated in presence of (C) albumin $(+\mathrm{Bic}+\mathrm{BSA})$ and in presence of (D) ORP-1 (+Bic+ORP-1) are included to show the shift in the amount of sperm cells with their acrosome reaction induced by ORP-1 compared to albumin. The left population is acrosome intact and the right population is acrosome reacted as depicted with the line connector arrows to A. * indicate a significant increase of effects compared to -BicBSA (in all comparisons $\mathrm{P}<0.05$ ).

Fig. 9 Reduction of cellular cholesterol and desmosterol levels and cellular oxysterol levels after incubation with sterol interacting proteins. A) Oxysterol binding proteins ORP-1 and ORP-2 are more effective in reducing oxysterols from bicarbonate activated sperm than albumin and MBCD. The total amount of oxysterols indicated is relative to the amount of oxysterols formed in absence of oxysterol interacting molecules (-Bic-BSA $=100 \%$ conform Fig. 1). B) The cellular levels of cholesterol and desmosterol (total amount of both sterols indicated here data for desmosterol and cholesterol separately see Supplemental Figure S1) are reduced by albumin (BSA) in a bicarbonate specific manner which can be blocked by vitamin $\mathrm{E}$ (vitE). MBCD lowers both free sterols to lesser extent but in a bicarbonate independent manner. The ORP-1 and ORP-2 treatments did not lower the cellular levels of cholesterol or desmosterol. In all cases the molar ratio of cholesterol to desmosterol remained approximately 10:1. Nevertheless a slightly higher proportion of desmosterol was depleted by albumin and MBCD when compared to cholesterol (see Supplemental Figure S1). Mean values \pm SEM are indicated, $n=3$. * indicate a significant effect when compared to $+\mathrm{Bic}-\mathrm{BSA} ; * *$ indicate a more pronounced effect when compared to $+\mathrm{Bic}+\mathrm{BSA}$ (in all comparisons $\mathrm{P}<0.05$ ). For distribution of oxysterol species cholesterol and desmosterol in the cell pellet and the supernatant of boar and mouse sperm specimen after treatment see Table 2 .

Fig. 10 Effects of MBCD-mediated sterol depletion on sperm activation and in vitro fertilization. A) In the absence of bicarbonate (-Bic) MBCD induced PY-20 labelling in the 
concentration rage of $0.5-10 \mathrm{mM} \mathrm{MBCD}$ used and with maximal effect at $2 \mathrm{mM}$. In the presence of bicarbonate (+Bic) a similar maximal response was seen at $0.5 \mathrm{mM}$ while the higher doses of MBCD were still but less effective. B) MBCD only induced hyper-activated sperm motility at 2 $\mathrm{mM}$ dose in the absence of bicarbonate (-Bic) but had no effects at lower or higher dose. In the presence of bicarbonate only marginal increase of hyper-activated sperm motility was noted at lower doses of MBCD ( 0.5 or $2 \mathrm{mM})$, whereas the highest dose of $10 \mathrm{mM} \mathrm{MBCD}$ had an adverse effect and inhibited hyper-activated sperm motility. (C) MBCD-mediated sterol depletion inhibits in vitro fertilization and induces oocyte degeneration. Increasing amounts of MBCD in albumin free bicarbonate enriched incubation media did not result in high IVF rates. Only at the dose of $2 \mathrm{mM}$ MBCD approx. 30 percent of the oocytes became fertilized which is a relatively poor figure when compared to IVF rates obtained with albumin (see Fig. 7). A dosedependent degeneration of porcine oocytes was noted when IVF was performed in bicarbonate enriched media with inclusion of MBCD. The data represent mean $\pm \mathrm{SEM}, \mathrm{n}=3$, per experiment and for $\mathbf{C}$ in each incubation type at least 20 oocytes were used. * indicate a significant effect when compared to $0 \mathrm{mM} \mathrm{MBCD}(\mathrm{P}<0.05)$.

Fig. 11 Schematic presentation of bicarbonate induced ROS dependent oxysterol loading and removal of excessive oxysterols. These two processes induce signs of sperm capacitation which does not depend on removal of cholesterol and desmosterol as has been shown by the use of ORPs. However, the formation of oxysterols allows a downstream depletion of cholesterol and desmosterol by BSA which is required for in vitro fertilization (IVF). Indicated as a dashed line is the action of MBCD causing a completely bicarbonate, ROS, oxysterol independent cholesterol and desmosterol removal from sperm. Although under very small concentration range MBCD this can give moderately low in vitro fertilization rates these same concentrations leads to very substantial oocyte degeneration. 
TABLE 1. Formation and albumin-dependent extraction of oxysterols in boar and mouse sperm before and after in vitro capacitation.

\begin{tabular}{lccc}
\hline & \multicolumn{3}{c}{ Incubation } \\
\cline { 2 - 4 } & $0 \mathrm{~h}$ & $2 \mathrm{~h}(-$ albumin $)$ & $2 \mathrm{~h}(+$ albumin $)$ \\
\hline Boar semen & & & \\
Oxysterol/cholesterol (\%) & $0.05 \pm 0.02$ & $0.82 \pm 0.16^{\mathrm{a}}$ & $0.94 \pm 0.20^{\mathrm{a}}$ \\
Oxysterol in supernatant (\%) & $4 \pm 2$ & $8 \pm 3$ & $60 \pm 18^{\mathrm{a}}$ \\
Mouse semen & & & \\
Oxysterol/cholesterol (\%) & $0.14 \pm 0.08$ & $1.03 \pm 0.29^{\mathrm{a}}$ & $1.51 \pm 0.60^{\mathrm{a}}$ \\
Oxysterol in supernatant (\%) & $8 \pm 3$ & $11 \pm 4$ & $72 \pm 12^{\mathrm{a}}$ \\
\hline
\end{tabular}

Mean values \pm SD are provided, data obtained from 4 boars and 4 mice, using three individual ejaculates for each boar $(n=4, r=3)$ or aspiration of 2 mouse epididymi $(n=4, r=2)$. The relative amount of total oxysterol formed from cholesterol $(100 \%)$ is indicated as well as the distribution of the formed oxysterols $(100 \%$ in semen) which was recovered in the supernatant after centrifugation. The remaining proportion of the oxysterols where present in the sperm pellet. For partition of oxysterol species see after capacitation in presence of albumin see Table 2. Data indicated with ${ }^{\text {a }}$ differed significantly from the $0 \mathrm{~h}$ condition. Similar data were reported for bovine sperm previously [4]. 
TABLE 2. The relative amount of cholesterol, desmosterol and oxysterol species extracted by albumin from in vitro capacitating boar and mouse sperm cells.

\begin{tabular}{lcc}
\hline \multirow{2}{*}{ Component } & \multicolumn{2}{c}{ Relative amount in supernatant } \\
\cline { 2 - 3 } & Boar semen & Mouse semen \\
\hline 3,5,6-Trihydroxycholesterol & $71 \pm 11 \%$ & $74 \pm 17 \%$ \\
7-Hydroxycholesterol & $59 \pm 14 \%$ & $75 \pm 14 \%$ \\
7-Ketocholesterol & $62 \pm 13 \%$ & $80 \pm 16 \%$ \\
22-Ketocholesterol & $55 \pm 10 \%$ & Not detected \\
25-Hydroxycholesterol & $56 \pm 13 \%$ & $76 \pm 17 \%$ \\
5,6 $\beta$-Epoxycholesterol & $38 \pm 7 \%$ & $53 \pm 13 \%$ \\
5,6 $\alpha$-Epoxycholesterol & $35 \pm 7 \%$ & $41 \pm 9 \%$ \\
Desmosterol & $34 \pm 5 \%$ & $38 \pm 5 \%$ \\
Cholesterol & $38 \pm 5 \%$ & $37 \pm 3 \%$ \\
\hline
\end{tabular}

The mean percentage \pm SD from the total of each component in semen that is recovered in the supernatant after a $2 \mathrm{~h}$ in vitro capacitation incubation $(+\mathrm{Bic},+\mathrm{BSA})$ is expressed $(\mathrm{n}=3)$. 
TABLE 3. Effects of bicarbonate and membrane antioxidants during in vitro capacitation of mouse sperm.

\begin{tabular}{lcccc}
\hline Treatment & - Bic+BSA & + Bic+BSA & +Bic+BSA+VitE & + Bic+BSA+VitA \\
\hline Hyperactivated motility & $4 \pm 3 \%$ & $48 \pm 14 \%^{\mathrm{a}}$ & $14 \pm 5 \%{ }^{\mathrm{b}}$ & $6 \pm 4 \%^{\mathrm{b}}$ \\
PY-20 positive in tail & $3 \pm 2 \%$ & $52 \pm 19 \%^{\mathrm{a}}$ & $12 \pm 8 \%{ }^{\mathrm{b}}$ & $7 \pm 2 \%^{\mathrm{b}}$ \\
Membrane intact & $93 \pm 4 \%$ & $78 \pm 12 \%$ & $92 \pm 5 \%$ & $89 \pm 6 \%$ \\
\hline
\end{tabular}

Mean $\%$ of sperm cells \pm SD with indicated properties after a $2 \mathrm{~h}$ incubation $(\mathrm{n}=3, \mathrm{r}=2)$.

${ }^{a}$ Indicates a significant difference induced by bicarbonate (+Bic+BSA versus $\left.-\mathrm{Bic}+\mathrm{BSA}\right)$.

${ }^{\mathrm{b}}$ Indicates a significant inhibition of the bicarbonate induced effects by inclusion of hydrophobic antioxidants. Note that PY20 labeling was in some sperm cells present in the cytoplasmic droplets and/or in the post-acrosomal head region. Although the $\%$ of such aberrant PY20 labeling patterns was $<5 \%$ it was invariable between samples (not induced by bicarbonate nor inhibited by hydrophobic anti-oxidants). For all samples the overall amount of motile sperm cells was $>75 \%$. 
Figure 1

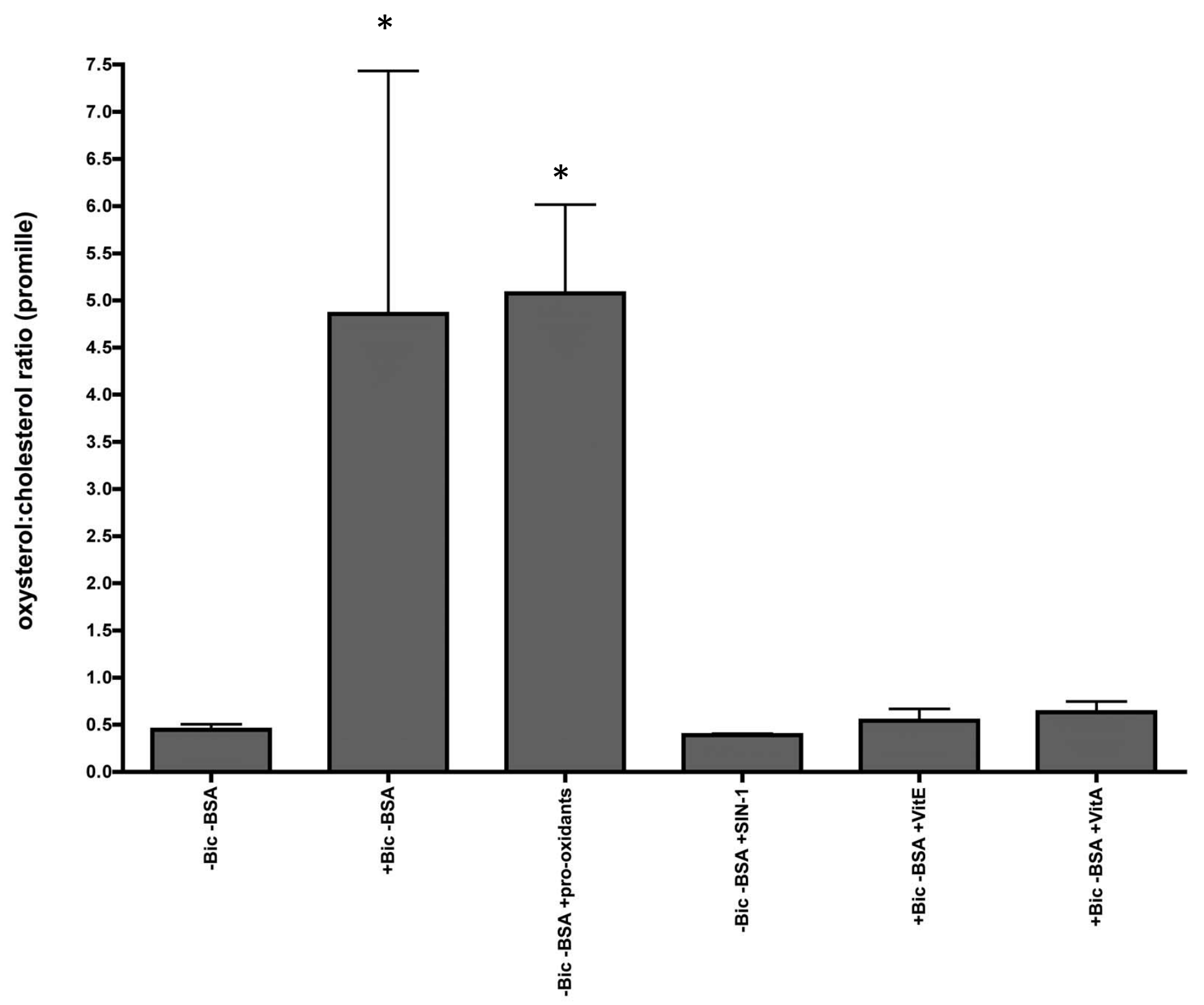


Figure 2

-Bic
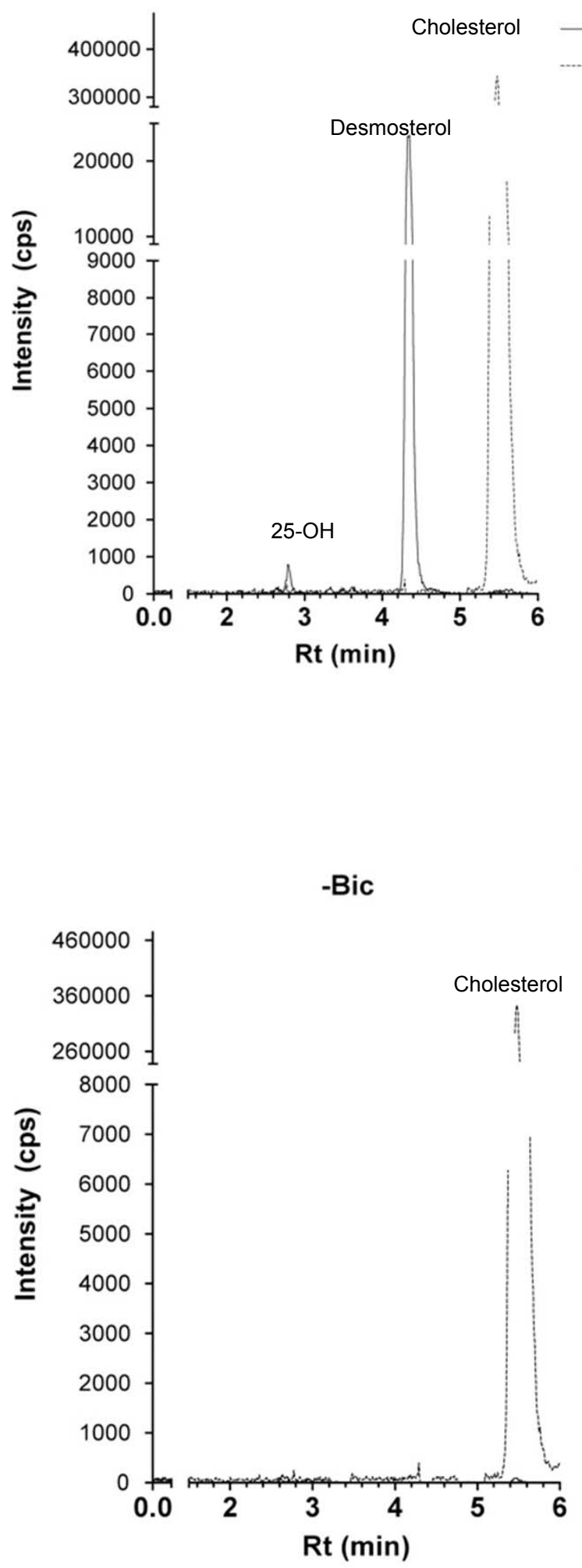

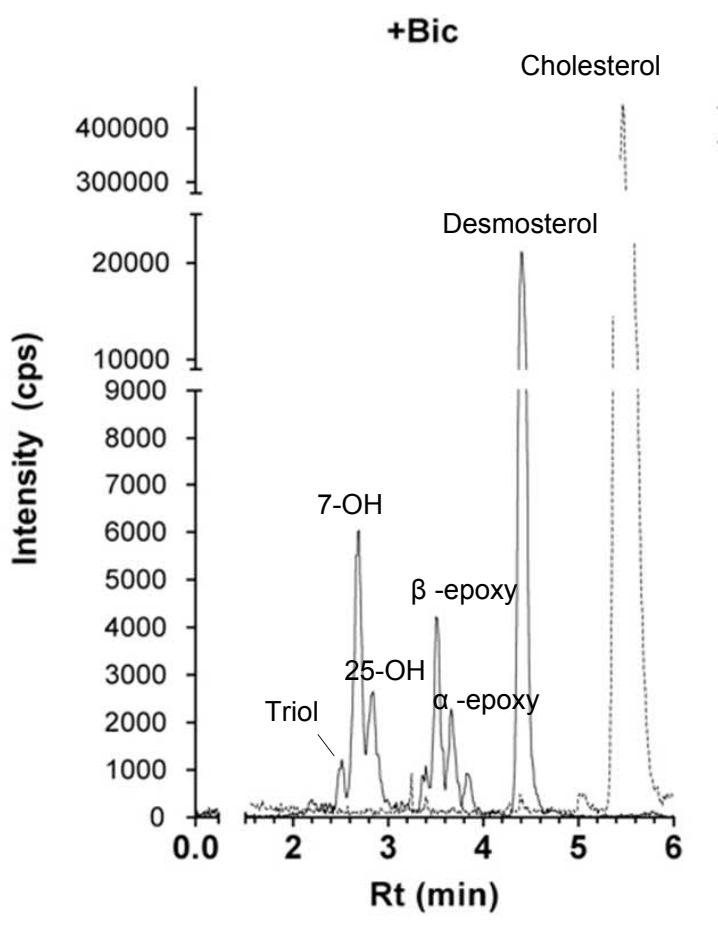

$-401 \mathrm{~m} / \mathrm{z}$

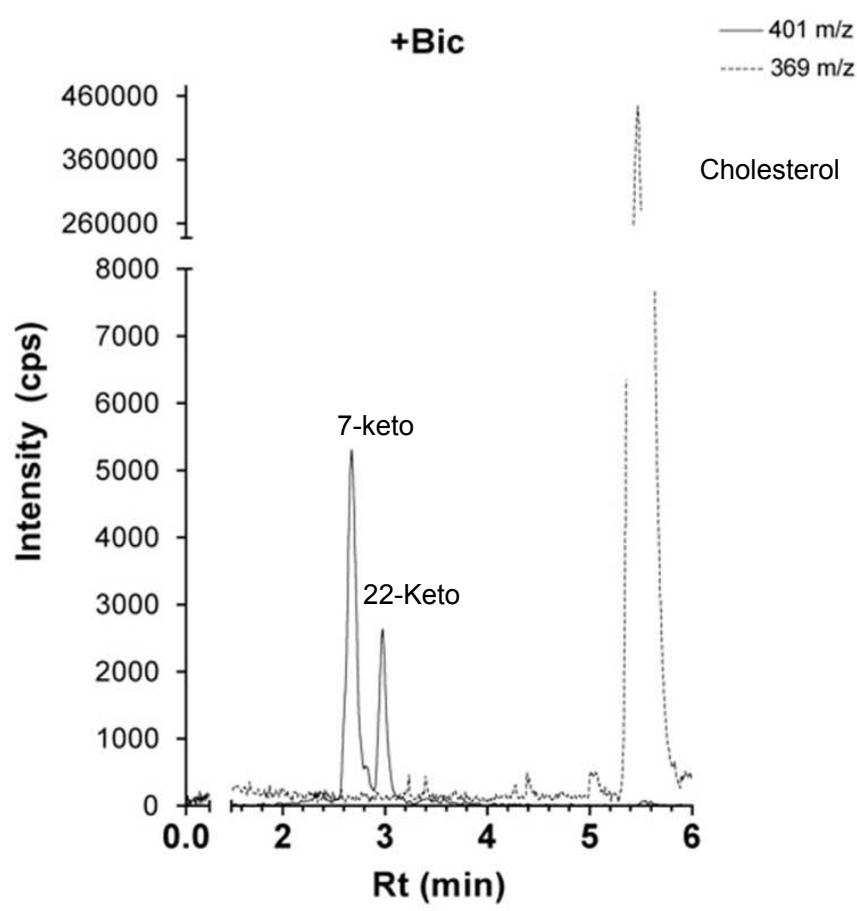




\section{Figure 3}

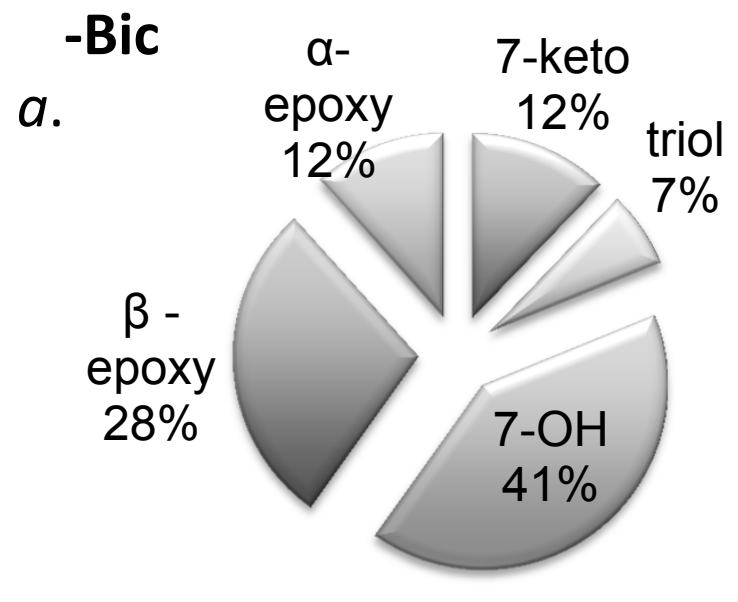

$0.5 \pm 0.1 \mathrm{pmol} / 10^{6}$ cells

\section{+Bic+BSA+VitE a-}

$a$.

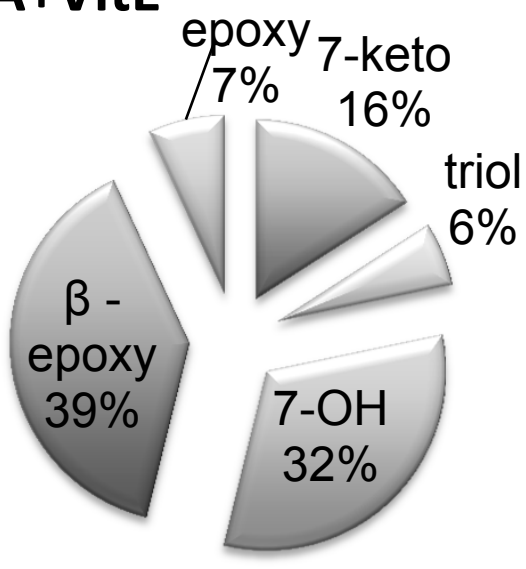

$0.7 \pm 0.4 \mathrm{pmol} / 10^{6}$ cells

\section{-Bic-BSA+pro-oxidants}

$b$.

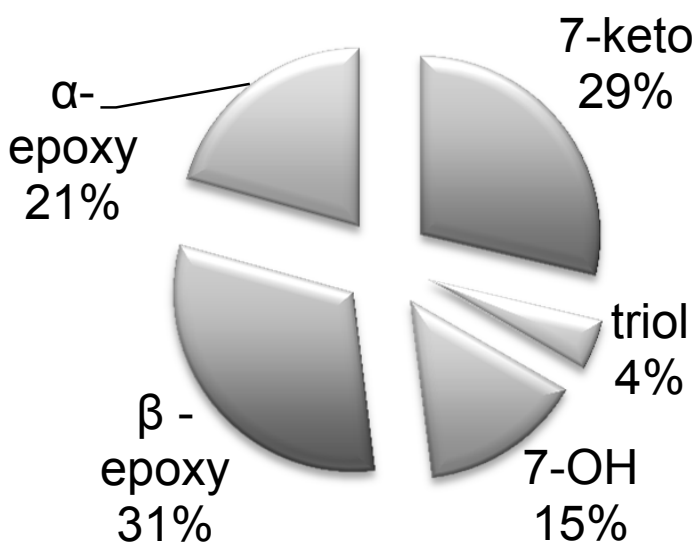

$10.1 \pm 4.1 \mathrm{pmol} / 10^{6}$ cells

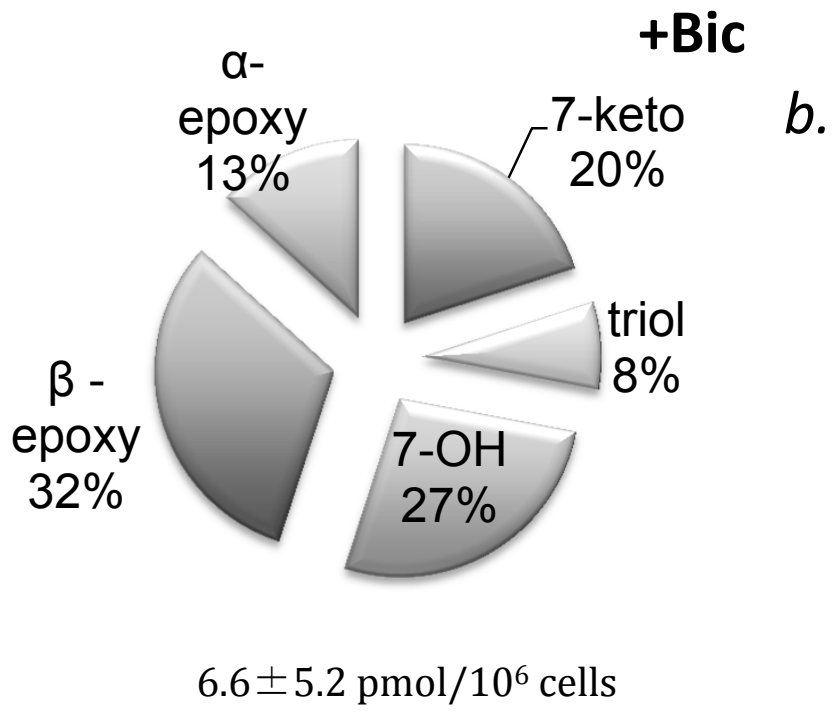

+ Bic+BSA

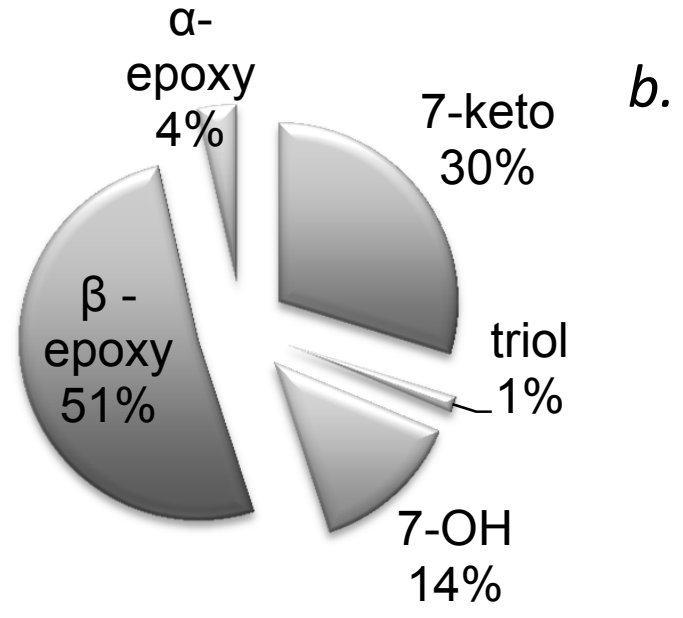

$1.8 \pm 2.1 \mathrm{pmol} / 10^{6}$ cells

+Bic+BSA+pro-oxidants

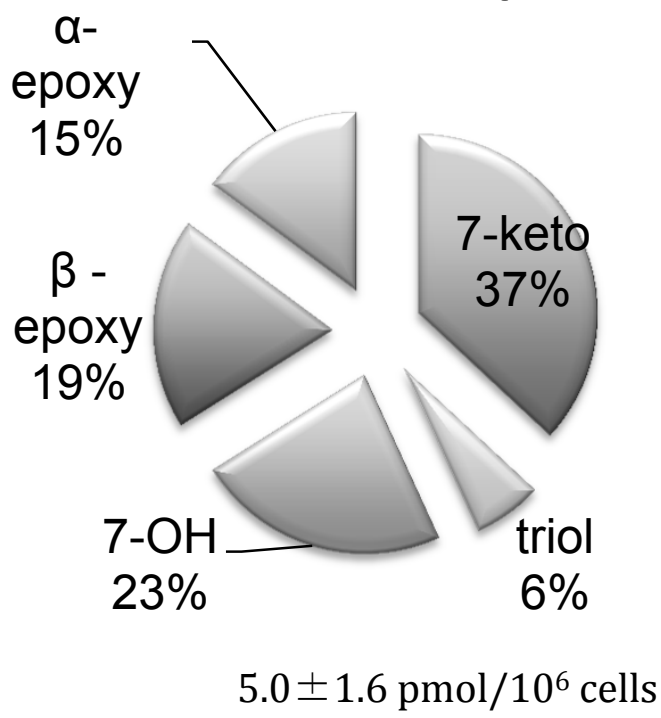


Figure 4
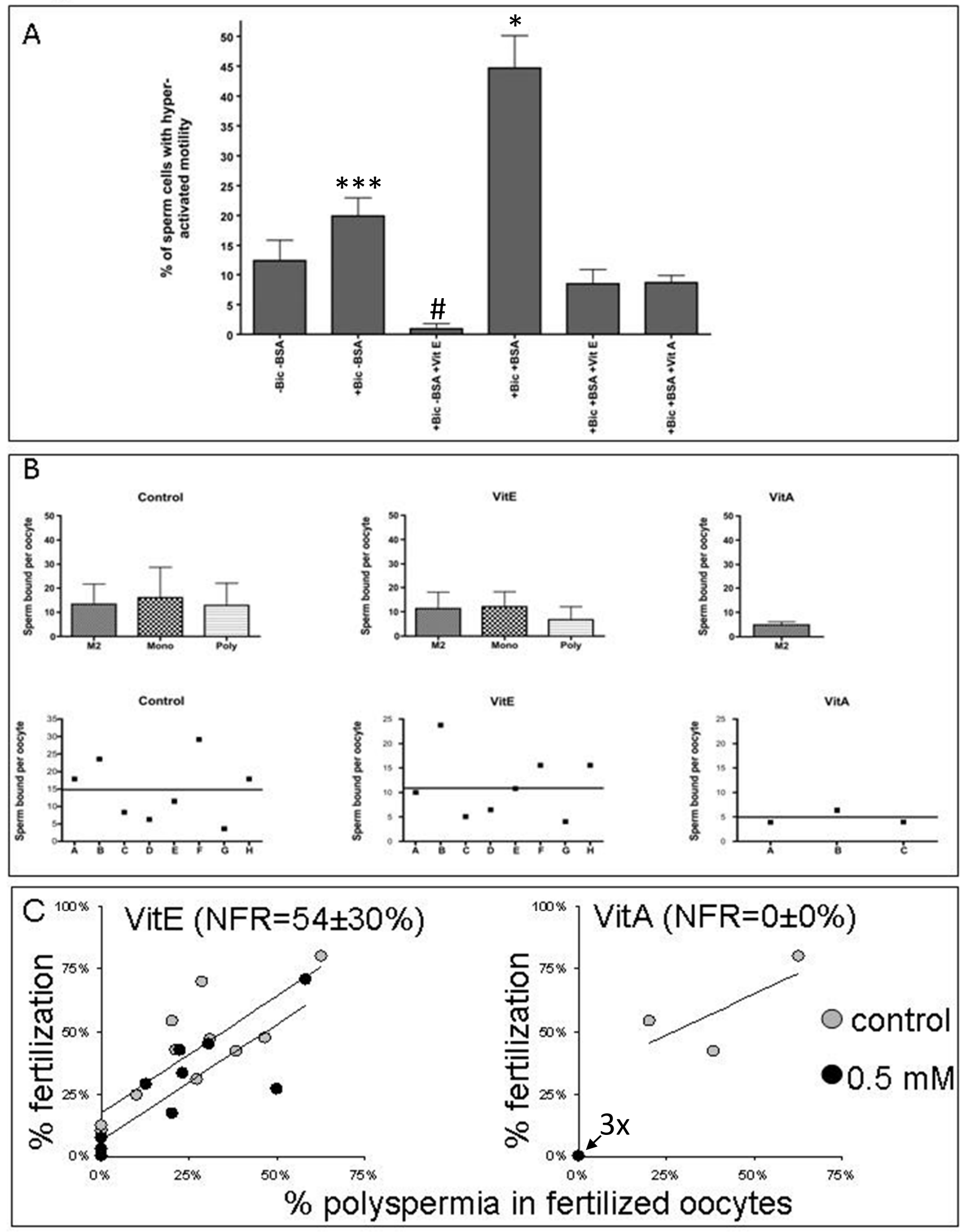
Figure 5
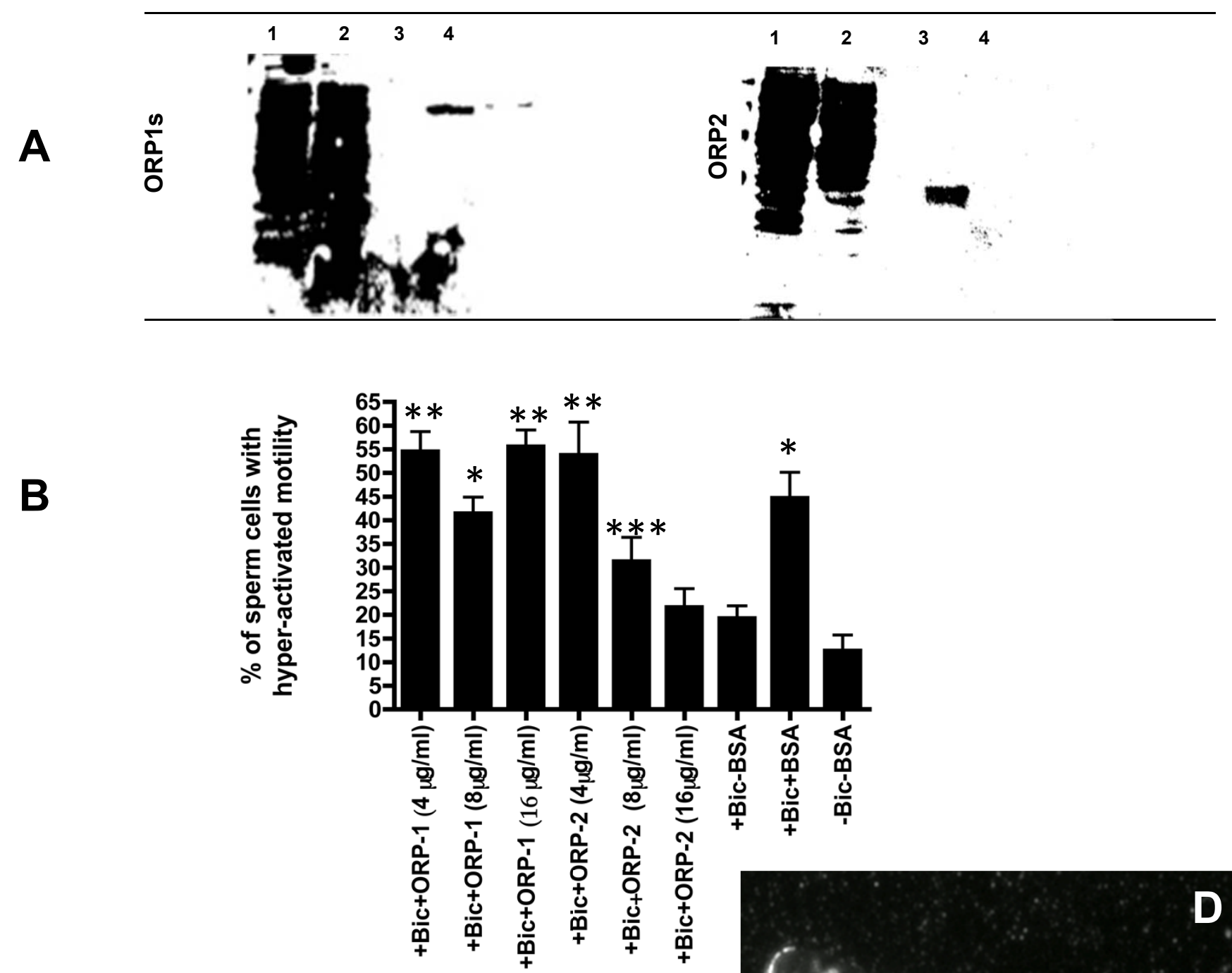

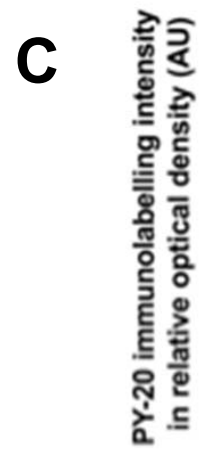
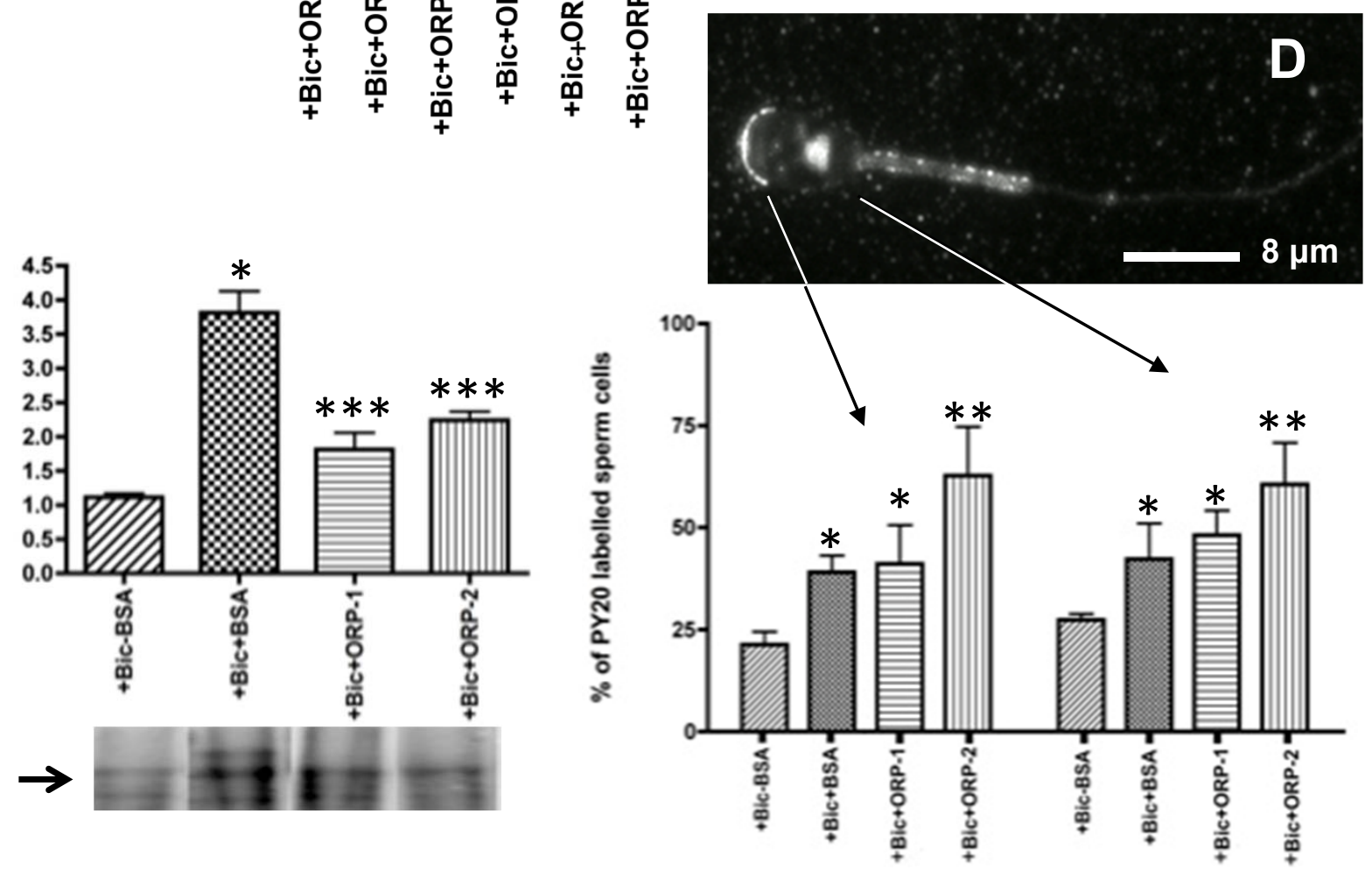

Apical area

Equatorial 
Figure 6

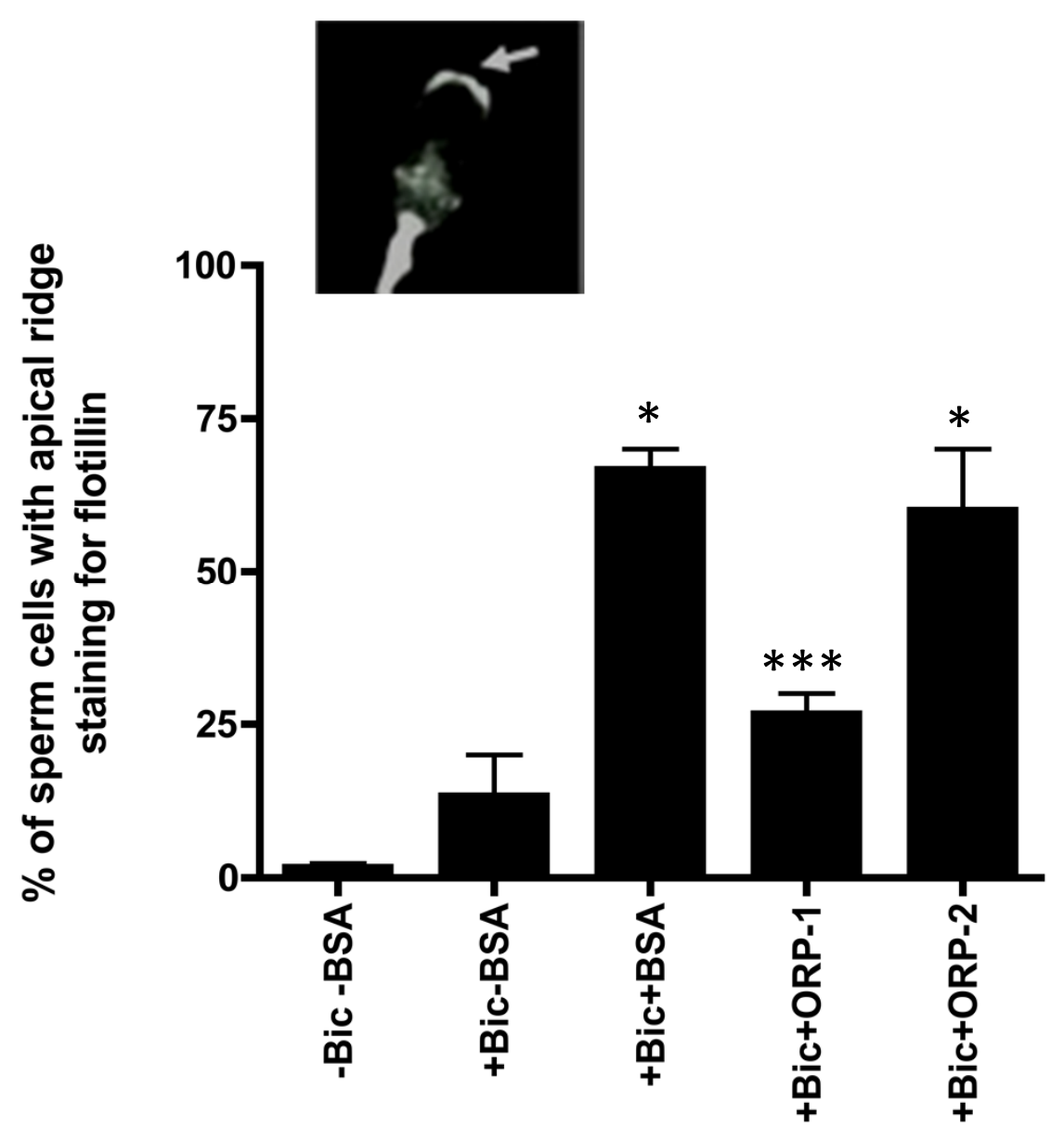


Figure 7

A

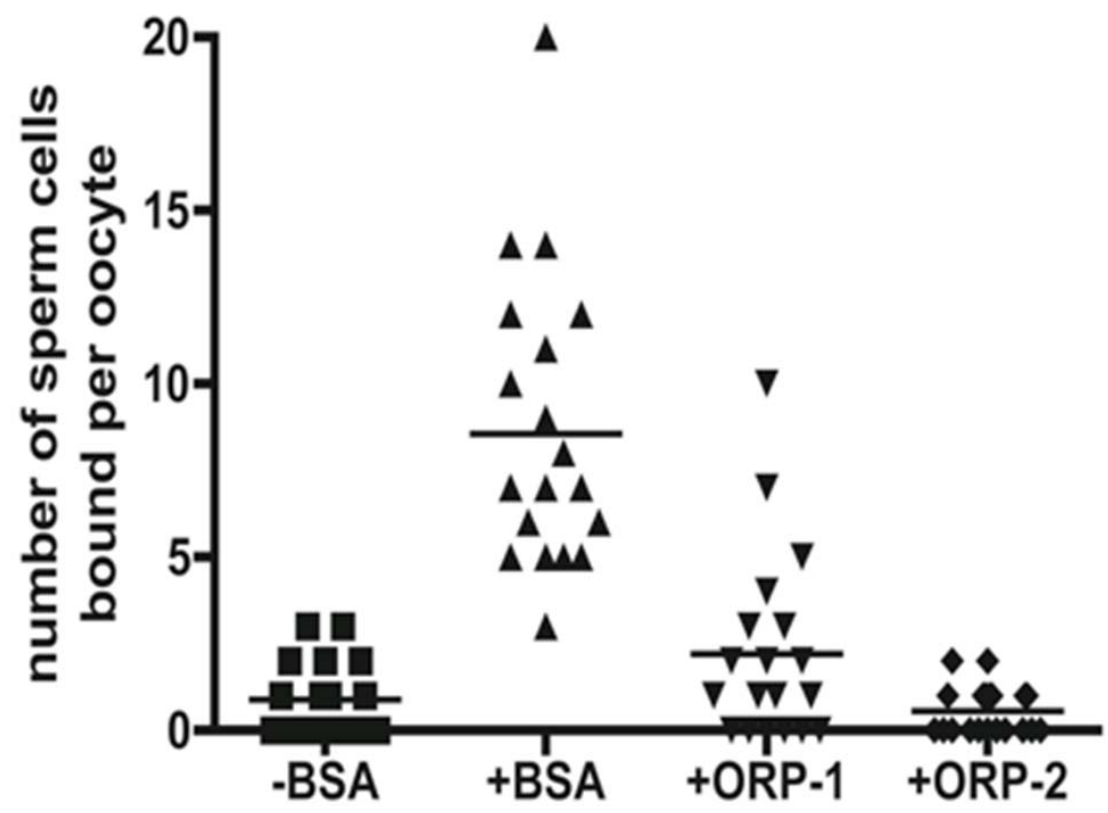

B

\begin{tabular}{|c|c|c|c|c|c|c|c|c|c|c|c|}
\hline \multirow[b]{2}{*}{ Germinal Vesicle } & \multicolumn{3}{|c|}{$-B S A$} & \multicolumn{3}{|c|}{$+B S A$} & \multicolumn{3}{|c|}{ +ORP-1 } & \multicolumn{2}{|c|}{ +ORP-2 } \\
\hline & 0.0 & \pm & 0.0 & 0.0 & \pm & 0.0 & 1.1 & & 2.0 & 2.1 & \pm 1.9 \\
\hline Metaphase I & 1.9 & \pm & 2.7 & 0.0 & \pm & 0.0 & 3.7 & \pm & 3.6 & 3.6 & \pm 4.0 \\
\hline Metaphase II & 91.4 & \pm & 1.3 & 31.0 & \pm & 8.5 & 86.8 & \pm & 10.2 & 85.2 & \pm 12.7 \\
\hline Fertilized & 2.4 & \pm & 3.4 & 60.8 & \pm & 6.2 & 3.5 & \pm & 3.6 & 4.1 & \pm 4.4 \\
\hline Degenerated & 4.3 & \pm & 0.6 & 8.1 & \pm & 6.4 & 4.9 & \pm & 5.4 & 4.9 & \pm 8.5 \\
\hline
\end{tabular}

C
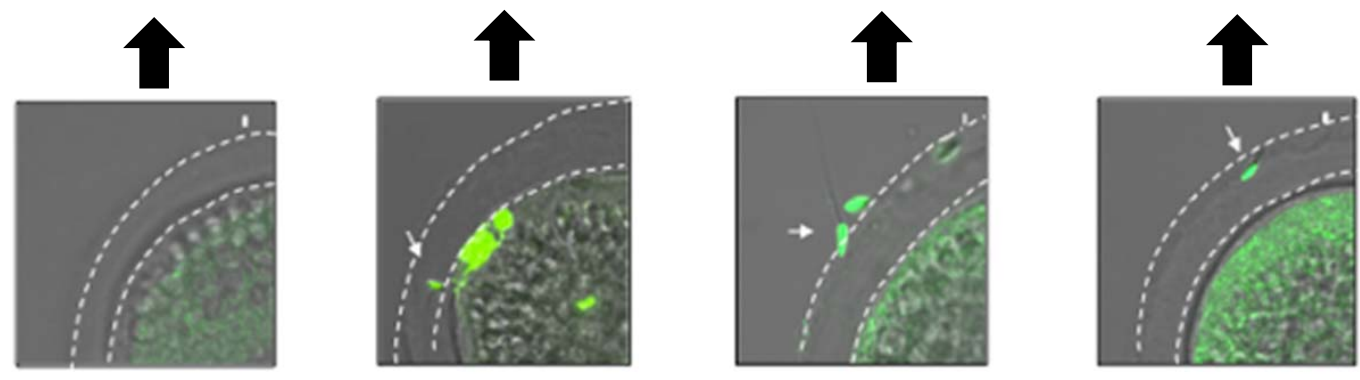
Figure 8

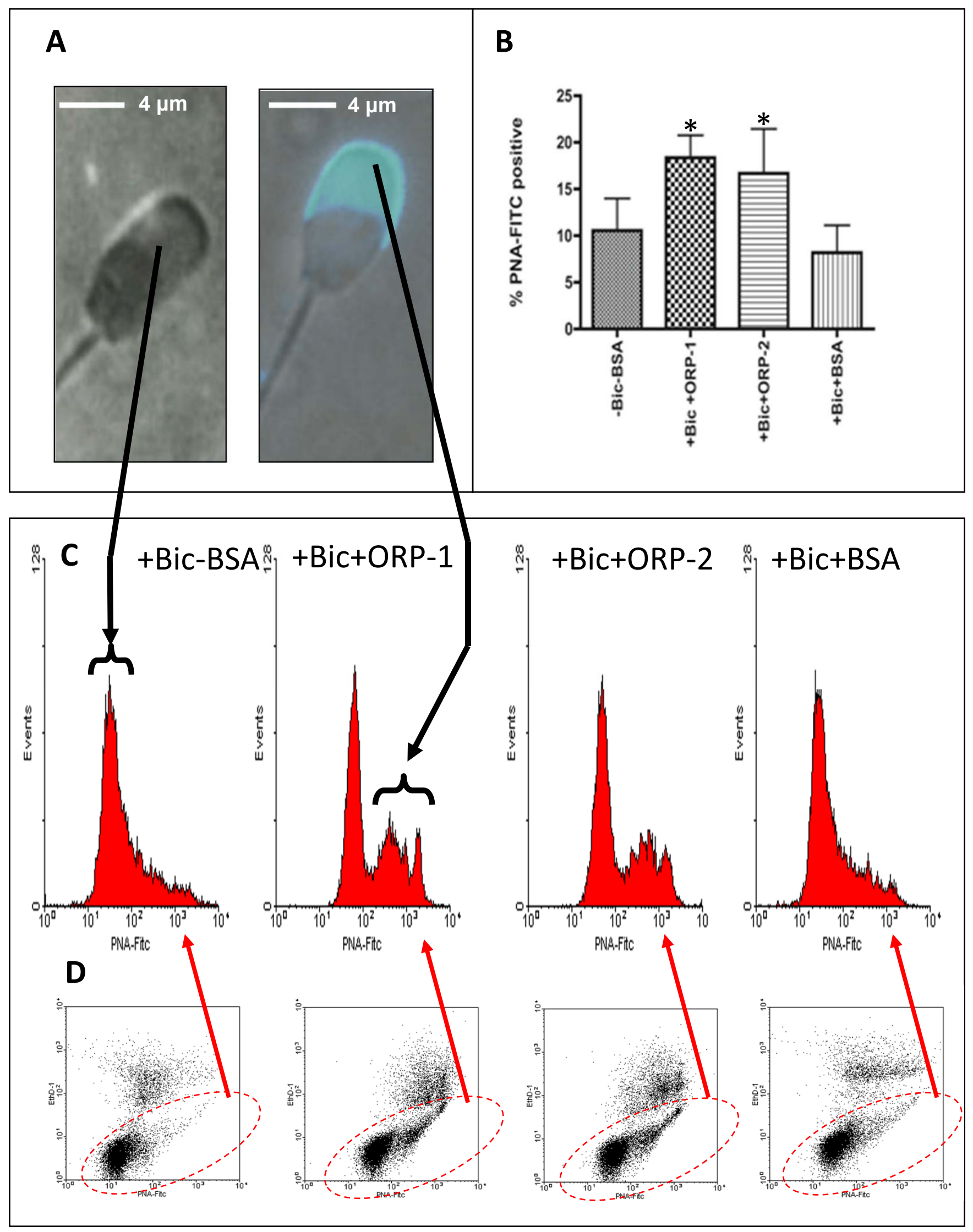


Figure 9

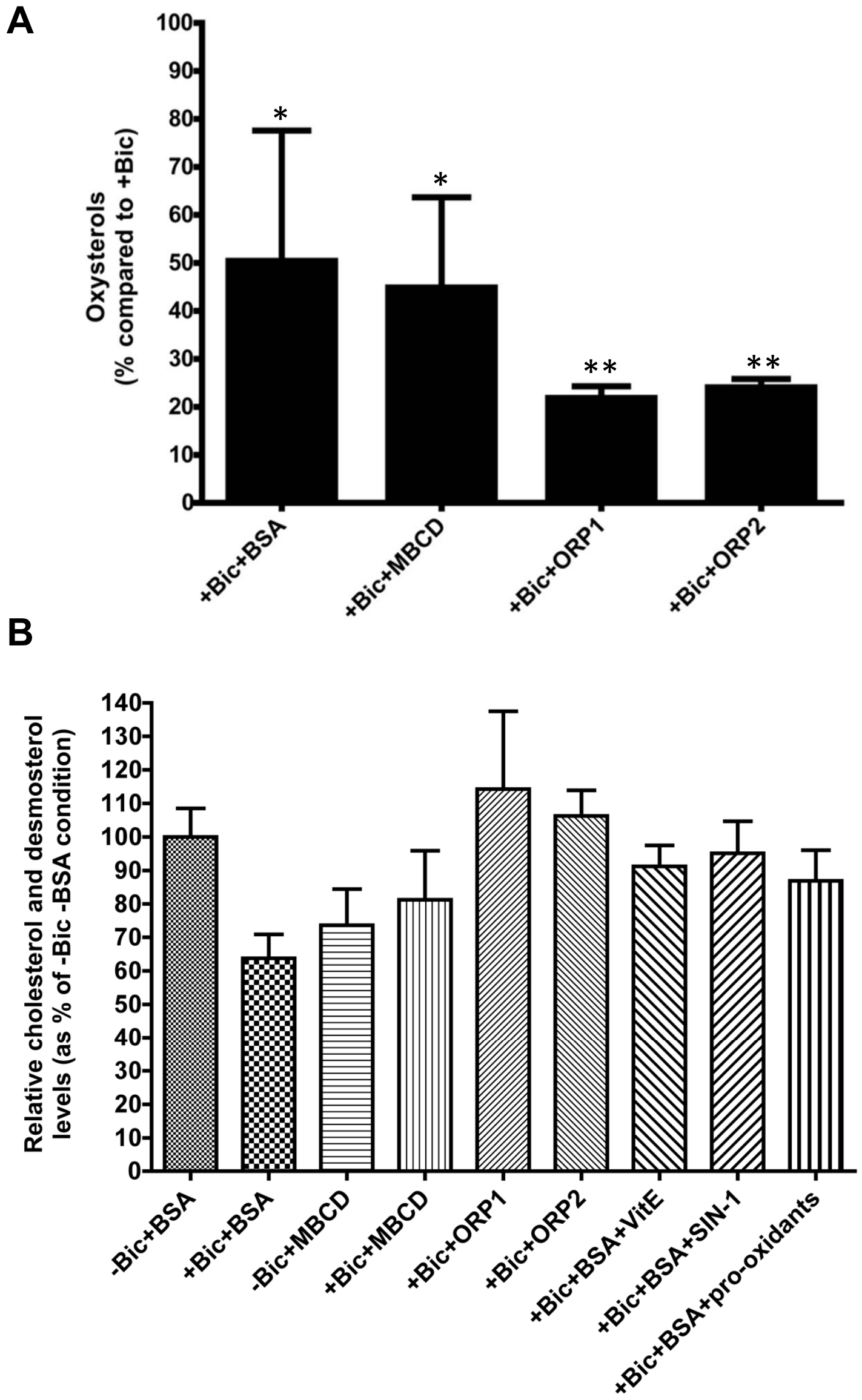


Figure 10
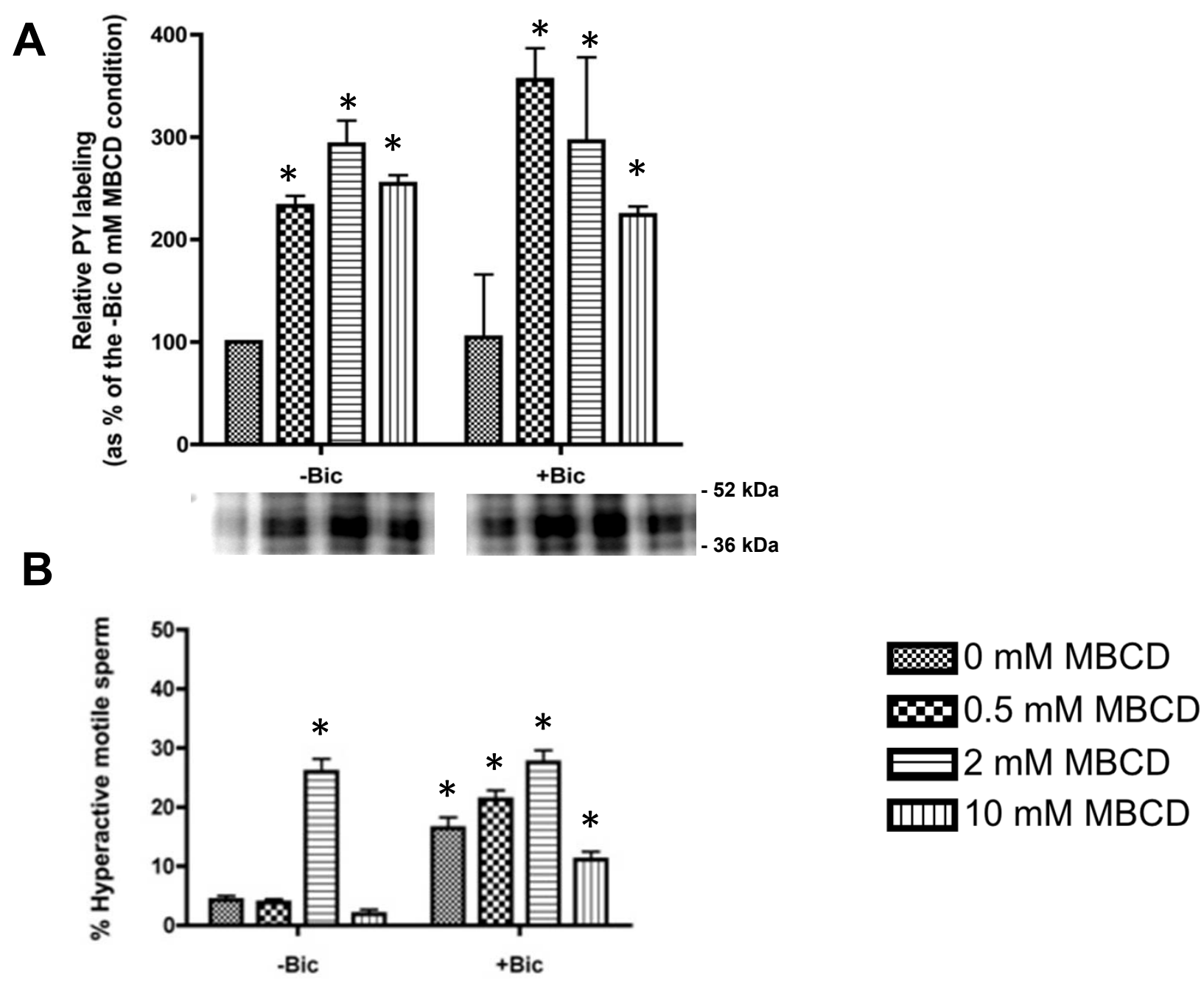

C

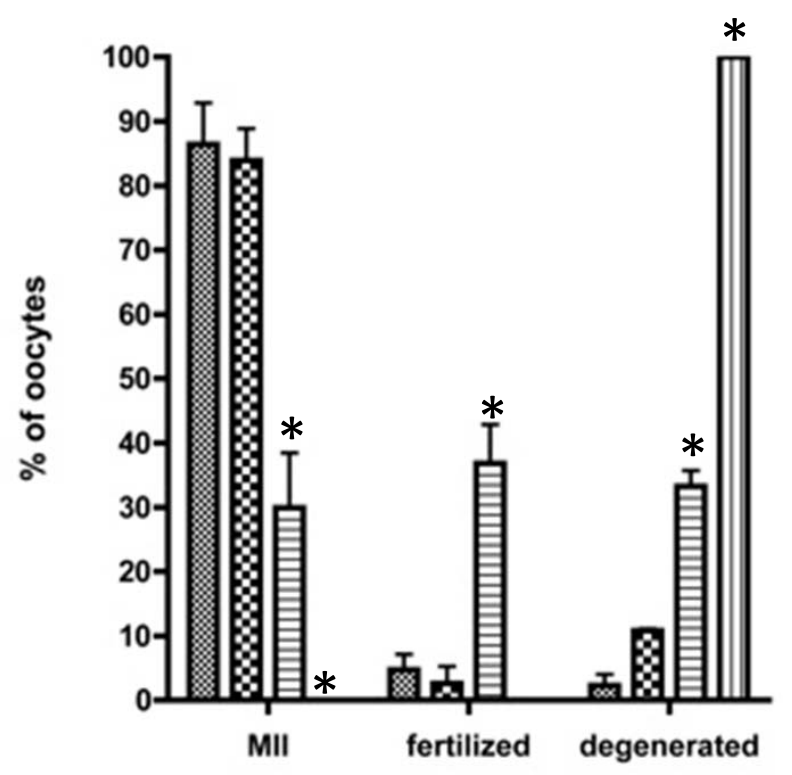


Figure 11

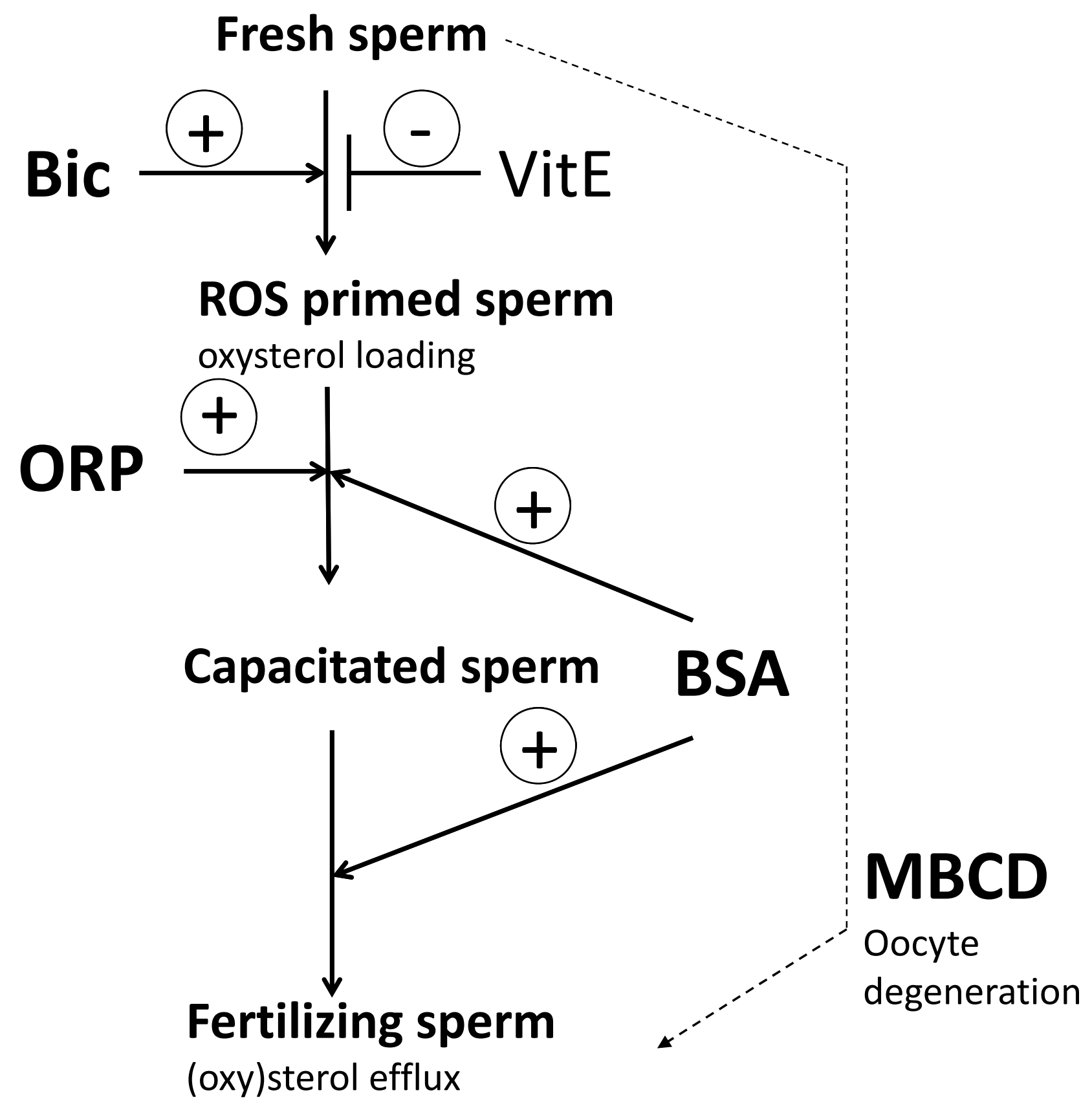

